\title{
Metapopulation Structure of Diatom-associated Marine Bacteria
}

2

$$
\text { Liping } \mathrm{Qu}^{1,2^{\wedge}} \text {, Xiaoyuan Feng }{ }^{3,2^{\wedge}} \text {, Yuerong } \mathrm{Chen}^{1} \text {, Lingyu } \mathrm{Li}^{1} \text {, Xiaojun Wang }{ }^{3,2} \text {, Zhong } \mathrm{Hu}^{1,4} \text {, }
$$

$$
\text { Hui Wang }{ }^{1,4^{*}} \text {, Haiwei } \text { Luo }^{3,2^{*}}
$$

${ }^{1}$ Biology Department, College of Science, and Guangdong Provincial Key Laboratory of Marine Biotechnology, and Institute of Marine Sciences, Shantou University, Shantou, China ${ }^{2}$ Shenzhen Research Institute, The Chinese University of Hong Kong, Shenzhen, China ${ }^{3}$ Simon F. S. Li Marine Science Laboratory, School of Life Sciences and State Key Laboratory of Agrobiotechnology, The Chinese University of Hong Kong, Shatin, Hong Kong SAR ${ }^{4}$ Southern Marine Science and Engineering Guangdong Laboratory, Guangzhou, China

${ }^{\wedge}$ These authors contributed equally to this study.

\section{*Corresponding author:}

Haiwei Luo

School of Life Sciences, The Chinese University of Hong Kong

Shatin, Hong Kong SAR

Phone: (+852) 39436121

E-mail: hluo2006@gmail.com

Hui Wang

Biology Department, College of Science, Shantou University

Shantou, China

Phone: (+86) 75486502721

E-mail:wanghui@ stu.edu.cn

\section{Running Title:}

Key words: Roseobacter, Sulfitobacter, diatom, phycosphere, population structure 


\section{Abstract}

Marine bacteria-phytoplankton interaction ultimately shapes ecosystem productivity. The

35 biochemical mechanisms underlying their interactions become increasingly known, yet how

36 these ubiquitous interactions drive bacterial evolution has not been illustrated. Here, we

37 sequenced genomes of 294 bacterial isolates associated with 19 coexisting diatom cells. These

38 bacteria constitute eight genetically monomorphic populations of the globally abundant

39 Roseobacter group. Six of these populations are members of Sulfitobacter, arguably the most

40 prevalent bacteria associated with marine diatoms. A key finding is that populations varying at

41 the intra-specific level have been differentiated and each are either associated with a single

42 diatom host or with multiple hosts not overlapping with those of other populations. These closely

43 related populations further show functional differentiation; they differ in motility phenotype and

44 they harbor distinct types of secretion systems with implication for mediating organismal

45 interactions. This interesting host-dependent population structure is even evident for demes

46 within a genetically monomorphic population but each associated with a distinct diatom cell, as

47 shown by a greater similarity in genome content between isolates from the same host compared

48 to those from different hosts. Importantly, the intra- and inter-population differentiation pattern

49 remains when the analyses are restricted to isolates from intra-specific diatom hosts, ruling out

50 distinct selective pressures and instead suggesting coexisting microalgal cells as physical barriers

51 of bacterial gene flow. Taken together, microalgae-associated bacteria display a unique

52 microscale metapopulation structure, which consists of numerous small populations whose

53 evolution is driven by random genetic drift. 
Since marine phytoplankton contribute one-half of global primary production (1) and

56 since heterotrophic bacterioplankton process $40-50 \%$ of the carbon fixed by marine

57 phytoplankton $(2,3)$, bacteria-phytoplankton interaction is an important process that ultimately

58 drives carbon cycling and regulates ecosystem productivity. The physical interface mediating

59 these ubiquitous interactions is a microzone of a few cell diameters immediately surrounding an

60 individual phytoplankton cell, which is termed as 'phycosphere' (4). In the phycosphere of

61 eukaryotic marine phytoplankton lineages (e.g., diatoms, dinoflagellates, coccolithophores, and

62 green pico-algae), bacterial communities are consistently dominated by a handful of taxa

63 including Rhodobacteraceae (mostly the Roseobacter group), Alteromonadaceae, and

64 Flavobacteriaceae $(4,5)$, and the bacterial community assembly at the phycosphere of a given

65 phytoplankton species is reproducible (6). These recurrent patterns in part result from the innate

66 ability of phytoplankton to modulate their bacteria consortia by secreting secondary metabolites

67 such as rosmarinic acid and azelaic acid released by a diatom species, which promote the

68 attachment and growth of certain roseobacters but suppress opportunistic bacteria (7). Another

69 important mechanism is the resource-based niche partitioning among these major bacterial

70 associates at nutrient-enhanced phycosphere $(8,9)$. For example, diatoms may use their abundant

71 metabolites such as 2,3-dihydroxypropane-1-sulfonate (DHPS) for targeted feeding of beneficial

72 symbionts among which roseobacters represent a dominant group (10).

Recent studies have revealed a greater diversity of the mechanisms underlying the

74 symbiosis between Roseobacter lineages and phytoplankton species than previously appreciated.

75 Some roseobacters such as Sulfitobacter spp., Ruegeria spp. and Dinoroseobacter spp. establish

76 mutualistic interactions with diatoms and microscopic green algae by providing growth factors

77 such as vitamins and indole-3-acetic acid (IAA) to phytoplankton hosts in exchange for labile 
organic matter (10-12), whereas others such as Sulfitobacter spp. are virulent to coccolithophores by releasing algicides (13). In another type of interaction, some roseobacters including Phaeobacter spp. and Dinoroseobacter spp. each act initially as a mutualist and later as a

81 parasite of coccolithophores and dinoflagellates, respectively $(14,15)$. An important implication

82 from these studies is that closely related roseobacters (e.g., members of Sulfitobacter) may

83 employ different mechanisms to interact with phytoplankton. This high diversity of roseobacter-

84 phytoplankton interaction therefore suggests that the phycosphere may act as an effective barrier

85 of gene flow among symbiotic roseobacters associated with different phytoplankton cells,

86 leading to independent evolution of even closely related roseobacter populations in the

87 seemingly well mixed seawater.

88 To test this hypothesis, we sought to determine the population structure of roseobacters

89 colonizing the phycosphere of coexisting microalgal cells. A few environmental factors

90 including nutrient availability (16), interactions with phages (17) and phytoplankton or particles

91 (18) are known to drive roseobacter population differentiation. To single out diatom phycosphere

92 from other confounding factors that may drive roseobacter evolution, populations associated with

93 diatoms isolated from a single seawater sample were analyzed. We collected 1 L of seawater

94 from the Pearl River Estuary located at the northern boundary of the South China Sea, isolated

9545 diatom cells varying at the level of phylogenetic relatedness (Fig. 1A, Table S1), cultivated

96 over 850 roseobacters associated with 19 of these diatoms (Fig. 1A, Table S2), and sequenced

97294 genomes of these roseobacters (Table S3), among which six genomes are complete and

98 closed (Table S4) by additional sequencing with Nanopore (Supplemental Text 1.1-1.3). These

99 newly sequenced roseobacters comprise eight clades (Fig. 1B; see methods in Supplemental Text

100 1.4), among which six are related to three species of Sulfitobacter, a roseobacter genus most 
101 commonly found on diatoms (19). These include clade-2a and clade-2b related to Sulfitobacter

102 pseudonitzschiae, clade-2c related to $S$. geojensis, clade-2d, clade-2e1 and clade-2e2 related to $S$.

103 mediterraneus (Fig. 1B). The remaining clade-1 and clade-3 are related to Marivita

104 cryptomonadis and Ponticoccus sp. LZ-14, respectively, which are distantly related to

105 Sulfitobacter. Members within each clade share identical 16S rRNA genes, display whole-

106 genome average nucleotide identity (ANI) over 99.99\% (Fig. S1), and vary up to 45 non-

107 singleton single nucleotide polymorphisms (SNPs) in which the rare variant occurs in at least

108 two genomes (Table S5). Despite this genetic monomorphism, there is ample evidence that the

109 within-clade members are predominantly from the environment rather than a result of clonal

110 replications during the laboratory cultivation of the diatom cells, a required process prior to

111 roseobacter isolation (see Supplemental Text 2, Fig. S2 and Table S5).

112 A key finding is that closely related roseobacter populations associated with different

113 diatom cells are often genetically differentiated (see methods in Supplemental Text 1.6). This is

114 clearly supported by populations diverged at different phylogenetic depths. The two clades

115 (clade-2a and clade-2b) related to S. pseudonitzschiae share 94.16\% ANI (Fig. S1), which is at

116 the boundary (95\% ANI) delineating a distinct species (20). The SNP density within each clade

117 is extremely low across the whole genome but becomes very high between the clades (Fig. S3),

118 indicating that these two clades each have fixed distinct alleles and are thus genetically isolated.

119 The clade-2a is composed solely of members isolated from Skeletonema menzelii 26 (abbreviated

120 as 'SM26'), whereas the clade-2b comprise members from five diatom cells of the class

121 Coscinodiscophyceae affiliated with three species including Skeletonema costatum (SC1 \&

122 SC33), Mediolabrus comicus (MC36 \& MC52), and Thalassiosira tenera (TT37), and one

123 diatom cell of a distantly related class Mediophyceae affiliated with the species Trieres chinensis 
124 (TC12) (Fig. 1B, Table S2). Among the three clades related to S. mediterraneus, clade-2e1 and

125 clade-2e 2 share $97.78 \%$ ANI, suggesting that they have not yet separated into two distinct

126 species. However, a dramatic increase in SNP density of between-clade comparisons compared

127 to the within-clade comparisons across the whole genome (Fig. S4A,B,C) indicates that these

128 two clades are under ongoing speciation. The clade-2d is more divergent, showing $89.34 \%$ ANI

129 to clade-2e1 and $89.51 \%$ ANI to clade-2e2, though it shares with the latter two clades very high

130 similarities (99.86\% and 99.93\%, respectively) at the 16S rRNA gene sequence. The SNP

131 density derived from the comparison between clade-2e1 and clade-2e 2 is much lower than when

132 each is compared to clade-2d (Fig. S4C,D,E), suggesting that the genomes are differentiated to a

133 greater extent when the phylogenetic depth becomes larger for these overall very closely related

134 clades. Importantly, these clades each have a distinct host range. Members of clade-2e1 are

135 exclusively associated with a single diatom host (SC5), whereas members of clade-2e2 are from

136 three hosts of the same diatom species (SC2, SC4 and SC7) and members of clade-2d are from

137 hosts of two different families (SC1 and MC36) (Fig. 1B, Table S2). Population differentiation

138 of the sampled clades is further supported by genome rearrangement in both chromosome and

139 plasmids, as shown by the more conserved gene order of within-clade members (Fig. S2)

140 compared to that of between-clade members (Fig. S5) for clade-2a and clade-2b. Multiple

141 genome rearrangement events were also observed between clade-2d and clade-2e2 (Fig. S6),

142 though within-clade comparisons cannot be made because no more closed genomes are available

143 in both clades.

144 Diatom-dependent differentiation is also evident from the more closely related

145 roseobacter demes associated with different hosts but sharing membership of the same clade.

146 Because of the genetic monomorphism at the core genomes (Table S5), the within-clade 
147 members do not show a reliable phylogenetic structure. We therefore turned to explore the

148 accessory genes which are shared by a subset of the genomes under comparison. A simple

149 clustering based on the presence and absence pattern of the accessory genes identified clusters

150 corresponding to distinct diatom hosts. In clade-2b, for example, members associated with SC33

151 largely constitute an independent cluster separated from members associated with other hosts

152 (Fig. 2A). Likewise, in clade-2c, members from Minutocellus polymorphus 20 (MP20) are

153 overall well separated from those associated with Thalassiosira rotula 60 (TR60) (Fig. 2B); in

154 clade-2e2, most members associated with SC2 are separated from those with other hosts (Fig.

155 2C); and in clade-3, members from SC2 and those from SC6 are generally clustered into two

156 separate groups (Fig. 2D). For the remaining clades (clade-2d \& clade-1) with members

157 associated with multiple hosts, host-dependent clustering is not obvious (Fig. S7).

We further identified important evidence for population differentiation at the functional

159 level. The secretion systems are well known to mediate bacteria-bacteria and bacteria-host

160 interactions. Interestingly, the presence and absence pattern of three secretion systems

161 differentiates the clade-2e1, clade-2e 2 and clade-2d related to $S$. mediterraneus (Table S6).

162 Specifically, the type VI secretion system (T6SS) transports effector proteins into both

163 prokaryotic and eukaryotic cells in a contact-dependent manner $(21,22)$. This system was

164 reported in only a few roseobacter lineages $(23,24)$. We showed an exclusive presence of a

165 T6SS gene cluster on the chromosome of clade-2d members. Another uncommon secretion

166 system in roseobacters, thus far only reported in the roseobacter species Marinovum algicola

167 (25), is the type II secretion system (T2SS), which promotes the release of folded proteins,

168 mainly extracellular enzymes such as proteases, lipases, phosphatases, and polysaccharide

169 hydrolases, to the extracellular milieu or displayed on the cell surface (26). We found an 
170 exclusive occurrence of a T2SS cluster on a plasmid of the clade- $2 \mathrm{~d}$ members. In terms of the

171 type IV secretion system (T4SS), the virB/D4 type secretes effector proteins and plasmid DNA

172 to target both bacteria and hosts (27-29), whereas the trb type transports plasmid DNA between

173 bacteria (29). While the $\operatorname{vir} B / D 4$ is commonly found among roseobacters $(30)$, the $t r b$ is rare in

174 these bacteria. Consistent with this pattern, a virB/D4 gene cluster was found in all three clades,

175 but a trb gene cluster was exclusively identified on the chromosome of clade-2e 2 members. In

176 the case of the $S$. pseudonitzschiae related clades, both clade-2a and clade-2b carry the virB/D4-

177 based T4SS, but they differ in copy numbers. The virB/D4 copy number difference was similarly

178 found between the three $S$. mediterraneus related clades, but a unique observation was that the

179 clade-2d members possess an additional copy on their chromosomes instead of the plasmids

180 where this type of secretion system usually locates. No other secretion systems were found in

181 clade-2a and clade-2b. Gene clusters encoding all secretion systems locate within the genomic

182 islands except the T6SS of clade-2d (Table S6), suggesting that roseobacter-diatom and/or

183 roseobacter-bacteria interactions are highly dynamic.

184 Since these secretion systems may mediate either pathogenic, or commensal, or

185 mutualistic relationships with hosts and/or other bacteria $(21,26,28,29,31,32)$, their

186 differential presence among the clades suggests that distinct clades may exert different and even

187 opposite physiological effects on the diatom hosts. This motivated us to set up experimental

188 assays (Supplemental Text 1.7) to compare the effects of co-culture of diatom and roseobacter,

189 the latter represented by each of the S. mediterraneus and S. pseudonitzschiae clades, on the

190 growth of the diatom. Among the 11 tested roseobacter isolates, eight significantly promoted the

191 growth of the diatom, whereas the remaining three did not significantly change the growth rate of

192 the diatom (Fig. S8). We did not observe consistent differences between closely related clades 
193 regarding their effects on the diatom growth (Fig. S8). While this assay was motivated by the

194 observation of clade-specific secretion systems, there is no direct link between the algal growth

195 change and the differential presence of the secretion systems in the bacterial symbionts.

196 Another important metabolic trait relevant to roseobacter-phytoplankton interaction is the

197 bacterial motility (33), which is differentially present among these related roseobacter clades.

198 Three phylogenetically distinct flagellar gene clusters (FGCs) designated as flal, fla2 and fla3

199 (Fig. 3A) have been identified in the Roseobacter group, and carrying any of them may enable

200 motility $(34,35)$. Among these, fla2 is present in a plasmid of the S. pseudonitzschiae related

201 clade-2e1, clade-2e2 and clade $2 \mathrm{~d}$, whereas flal was exclusively found on the chromosome of

202 clade-2e2 (Fig. 3A, Table S6; see methods in Supplemental Text 1.8). Despite the presence of

203 the flagellum-encoding gene clusters, the flagella were not detected by transmission electron

204 microscopy (Fig. 3B) and the motility phenotype was not observed under the experimental

205 condition (Fig. 3B; Supplemental Text 1.9). The lack of flagella and motility in these related

206 clades is likely due to inappropriate physicochemical conditions set in the laboratory experiment,

207 as temperature (36), $\mathrm{pH}(37)$, salinity (38) and metal ions (39) were demonstrated to induce the

208 expression of the FGC genes in other bacteria. In terms of the $S$. pseudonitzschiae related clade-

209 2a and clade-2b, they did not possess any type of FGC (Table S6), but both instead carry

210 homologs of two candidate gene clusters (i.e., type-IVb tight adherence pilus gene cluster; Table

211 S6) recently hypothesized to be responsible for dendritic motility (34). We showed that the

212 clade-2b members possess an additional copy located within a genomic island compared to

213 clade-2a members, consistent with the greater swimming and dendritic motilities observed in the

214 former (Fig. 3C). 
216 population differentiation in free-living prokaryotic lineages $(18,40-42)$. In the present study, we

217 provided evidence that closely related but genetically discrete populations of several sympatric

218 Roseobacter lineages each have a distinct diatom host range. The pattern of Sulfitobacter

219 mediterraneus related populations (clade-2e1, clade-2e2, clade-2d) is of particular interest. This

220 is because populations of this species varying at different stages of differentiation, including

221 those at the very beginning (i.e., within-clade demes differentiated only by accessory gene

222 content), at the middle (i.e., closely related clade under ongoing speciation), and at the

223 completion of speciation, were captured and each found to be associated with different hosts of

224 the same diatom species Skeletonema costatum. Since members of the same microalgal species

225 likely release a similar set of organic compounds to the phycosphere and impose other

226 physicochemical parameters (e.g., reactive oxygen species) at similar levels, the observed

227 differentiation of the symbiotic bacterial populations is less likely driven by ecological selection

228 imposed by differential exposure to different microalgal exudates. Instead, symbiotic bacteria

229 may be trapped in the phycosphere (43), leading to a reduced opportunity of recombination

230 between bacteria associated with different diatom cells compared to those within the same

231 phycosphere. This is a new mechanism of bacterial population differentiation in the pelagic

232 ocean and represents one of the few examples of population differentiation at the sympatric scale

233 due to physical barriers of gene flow. To put it in context, previous cases of bacterial population

234 differentiation in a sympatric pelagic environment were linked to ecological barriers of gene

235 flow, such as differentiated populations colonizing organic particles of different sizes (41) or

236 those inhabiting the bulk seawater versus phycosphere/particles (18). 
Our observation of subdivision of highly closely related populations each showing genetic monomorphism has important implications for understanding the population structure of

239 the diatom-associated symbiotic roseobacters. Similar population structure was previously

240 demonstrated in obligately host-dependent bacteria such as endosymbionts subjected to repeated

241 bottlenecks during transmissions to new hosts in small numbers of bacterial cells (44), and also

242 proposed for generalist marine bacteria such as Vibrio spp. which experience short bursts as a

243 result of intensive use of ephemeral resources like organic particles followed by dispersal and

244 colonization of new particles with low numbers of cells (45). These two known mechanisms lead

245 to the formation of "metapopulation structure", in which the population is divided into

246 subpopulations each colonizing a transient resource such as hosts and particles (45). Our results

247 suggest that the population structure of the diatom-associated roseobacters aligns well with the

248 metapopulation structure. Hence, phycosphere colonization represents a new mechanism leading

249 to bacterial metapopulation structure in the pelagic ocean. The exact processes leading to the

250 formation of metapopulation structure of these diatom-associated roseobacters remains unknown,

251 however. It could be a result of short burst owing to intensive use of the organic substrates

252 enriched in the phycosphere. It is also possible that bacteria-diatom associations have been

253 maintained at the evolutionary timescale, such that diatom host-dependent population

254 differentiation is evident even at the completion of speciation.

256 population size $\left(N_{e}\right)$ of the species (45), a key parameter in understanding the population genetic

257 mechanism underpinning biological evolution and defined as the size of an ideal population

258 carrying the same amount of the neutral genetic diversity as is observed in the real population

$259(45,46)$. Because $N_{e}$ is the inverse of the power of random genetic drift (45), the reduced $N_{e}$ of a 
260 bacterial species owing to the formation of metapopulation structure suggests an increased power

261 of genetic drift in driving the evolution of diatom-association roseobacters. As a consequence,

262 the diatom-associated roseobacter populations are predicted to more readily accept the

263 horizontally transferred genetic elements, which are often mildly deleterious owing to the selfish

264 propagation of most mobile genetic elements at the expense of cellular fitness (47) but may also

265 carry functional traits such as antimicrobial genes that increase competitive advantages of the

266 bacteria at the phycosphere. Given that diatoms and roseobacters are among the most abundant

267 phytoplankton and bacterial groups, respectively, in today's ocean (19), our findings have

268 important implications for biogeochemical cycles mediated by bacteria-phytoplankton

269 interactions.

270 It is important to clarify that while the concept of phycosphere has been adopted in this

271 and many other studies, there has been no direct experimental evidence for its occurrence

272 because of technological challenges in separating this microenvironment from the bulk seawater

273 (4). Prior studies established microalgal phycosphere as a hotspot of carbon and nutrient cycling

274 in the pelagic ocean. Here, we revealed that phycosphere of diatom cells may act as an effective

275 physical barrier of gene flow between nearly identical symbiotic roseobacters, thereby conferring

276 a new role of phycosphere in driving the evolution of pelagic marine bacteria. 


\section{References}

280 1. C. B. Field, M. J. Behrenfeld, J. T. Randerson, P. Falkowski, Primary production of the biosphere: integrating terrestrial and oceanic components. Science 281, 237-240 (1998).

2. F. Azam, T. Fenchel, J. G. Field, J. S. Gray, L. A. Meyer-Reil, F. Thingstad, The ecological role of water-column microbes in the sea. Marine Ecology Progress Series 10, 257-263 (1983).

3. J. J. Cole, S. Findlay, M. L. Pace, Bacterial production in fresh and saltwater ecosystems: A cross-system overview. Marine Ecology Progress Series 43, 1-10 (1988).

4. J. R. Seymour, S. A. Amin, J.-B. Raina, R. Stocker, Zooming in on the phycosphere: the ecological interface for phytoplankton-bacteria relationships. Nature Microbiology 2 , 17065 (2017).

5. A. Buchan, G. R. LeCleir, C. A. Gulvik, J. M. Gonzalez, Master recyclers: features and functions of bacteria associated with phytoplankton blooms. Nature Reviews Microbiology 12, 686-698 (2014).

6. J. Mönnich, J. Tebben, J. Bergemann, R. Case, S. Wohlrab, T. Harder, Niche-based assembly of bacterial consortia on the diatom Thalassiosira rotula is stable and reproducible. The ISME Journal 14, 1614-1625 (2020).

7. A. A. Shibl, A. Isaac, M. A. Ochsenkühn, A. Cárdenas, C. Fei, G. Behringer, M. Arnoux, N. Drou, M. P. Santos, K. C. Gunsalus, C. R. Voolstra, S. A. Amin, Diatom modulation of select bacteria through use of two unique secondary metabolites. Proceedings of the National Academy of Sciences 117, 27445-27455 (2020).

8. H. Fu, M. Uchimiya, J. Gore, M. Moran, Ecological drivers of bacterial community assembly in synthetic phycospheres. Proceedings of the National Academy of Sciences 117, 201917265 (2020).

9. F. X. Ferrer-González, B. Widner, N. R. Holderman, J. Glushka, A. S. Edison, E. B. Kujawinski, M. A. Moran, Resource partitioning of phytoplankton metabolites that support bacterial heterotrophy. The ISME Journal 15, 762-773 (2021).

10. B. P. Durham, S. Sharma, H. Luo, C. B. Smith, S. A. Amin, S. J. Bender, S. P. Dearth, B. A. S. Van Mooy, S. R. Campagna, E. B. Kujawinski, E. V. Armbrust, M. A. Moran, Cryptic carbon and sulfur cycling between surface ocean plankton. Proceedings of the National Academy of Sciences 112, 453-457 (2015).

11. S. A. Amin, L. R. Hmelo, H. M. van Tol, B. P. Durham, L. T. Carlson, K. R. Heal, R. L. Morales, C. T. Berthiaume, M. S. Parker, B. Djunaedi, A. E. Ingalls, M. R. Parsek, M. A. Moran, E. V. Armbrust, Interaction and signalling between a cosmopolitan phytoplankton and associated bacteria. Nature 522, 98-101 (2015).

12. M. B. Cooper, E. Kazamia, K. E. Helliwell, U. J. Kudahl, A. Sayer, G. L. Wheeler, A. G. Smith, Cross-exchange of B-vitamins underpins a mutualistic interaction between Ostreococcus tauri and Dinoroseobacter shibae. The ISME Journal 13, 334-345 (2019).

13. N. Barak-Gavish, M. J. Frada, C. Ku, P. A. Lee, G. R. DiTullio, S. Malitsky, A. Aharoni, S. J. Green, R. Rotkopf, E. Kartvelishvily, U. Sheyn, D. Schatz, A. Vardi, Bacterial virulence against an oceanic bloom-forming phytoplankter is mediated by algal DMSP. Science Advances 4, eaau5716 (2018).

14. I. Wagner-Dobler, B. Ballhausen, M. Berger, T. Brinkhoff, I. Buchholz, B. Bunk, H. Cypionka, R. Daniel, T. Drepper, G. Gerdts, S. Hahnke, C. Han, D. Jahn, D. Kalhoefer, H. Kiss, H.-P. Klenk, N. Kyrpides, W. Liebl, H. Liesegang, L. Meincke, A. Pati, J. 
Petersen, T. Piekarski, C. Pommerenke, S. Pradella, R. Pukall, R. Rabus, E. Stackebrandt, S. Thole, L. Thompson, P. Tielen, J. Tomasch, M. von Jan, N. Wanphrut, A. Wichels, H.

15. E. Segev, T. P. Wyche, K. H. Kim, J. Petersen, C. Ellebrandt, H. Vlamakis, N. Barteneva, Zech, M. Simon, The complete genome sequence of the algal symbiont Dinoroseobacter shibae: a hitchhiker's guide to life in the sea. The ISME Journal 4, 61-77 (2010).

J. N. Paulson, L. Chai, J. Clardy, R. Kolter, Dynamic metabolic exchange governs a marine algal-bacterial interaction. eLife 5, e17473 (2016).

16. Y. Sun, Y. Zhang, J. T. Hollibaugh, H. Luo, Ecotype diversification of an abundant Roseobacter lineage. Environmental Microbiology 19, 1625-1638 (2017).

17. E. C. Sonnenschein, K. F. Nielsen, P. D'Alvise, C. H. Porsby, J. Melchiorsen, J. Heilmann, P. G. Kalatzis, M. Lopez-Perez, B. Bunk, C. Sproer, M. Middelboe, L. Gram, Global occurrence and heterogeneity of the Roseobacter-clade species Ruegeria mobilis. The ISME Journal 11, 569-583 (2017).

18. X. Wang, Y. Zhang, M. Ren, T. Xia, X. Chu, C. Liu, X. Lin, Y. Huang, Z. Chen, A. Yan, H. Luo, Cryptic speciation of a pelagic Roseobacter population varying at a few thousand nucleotide sites. The ISME Journal 14, 3106-3119 (2020).

19. S. A. Amin, M. S. Parker, E. V. Armbrust, Interactions between diatoms and bacteria. Microbiology and Molecular Biology Reviews 76, 667-684 (2012).

20. C. Jain, L. M. Rodriguez-R, A. M. Phillippy, K. T. Konstantinidis, S. Aluru, High throughput ANI analysis of $90 \mathrm{~K}$ prokaryotic genomes reveals clear species boundaries. Nature Communications 9, 5114 (2018).

21. T. G. Sana, A. Hachani, I. Bucior, C. Soscia, S. Garvis, E. Termine, J. Engel, A. Filloux, S. Bleves, The second type VI secretion system of Pseudomonas aeruginosa strain PAO1 is regulated by quorum sensing and Fur and modulates internalization in epithelial cells. Journal of Biological Chemistry 287, 27095-27105 (2012).

22. J. M. Silverman, Y. R. Brunet, E. Cascales, J. D. Mougous, Structure and regulation of the type VI secretion system. Annual Review of Microbiology 66, 453-472 (2012).

23. Y. Zhang, Y. Sun, N. Jiao, R. Stepanauskas, H. Luo, Ecological genomics of the uncultivated marine Roseobacter lineage CHAB-I-5. Applied and Environmental Microbiology 82, 2100-2111 (2016).

24. A. J. Collins, M. S. Fullmer, J. P. Gogarten, S. V. Nyholm, Comparative genomics of Roseobacter clade bacteria isolated from the accessory nidamental gland of Euprymna scolopes. Frontiers in Microbiology 6, 123 (2015).

25. O. Frank, M. Göker, S. Pradella, J. Petersen, Ocean's Twelve: flagellar and biofilm chromids in the multipartite genome of Marinovum algicola DG898 exemplify functional compartmentalization. Environmental Microbiology 17, 4019-4034 (2015).

26. M. Nivaskumar, O. Francetic, Type II secretion system: A magic beanstalk or a protein escalator. Biochimica et Biophysica Acta (BBA) - Molecular Cell Research 1843, 15681577 (2014).

27. R. Fronzes, P. J. Christie, G. Waksman, The structural biology of type IV secretion systems. Nature Reviews Microbiology 7, 703-714 (2009).

28. K. Wallden, A. Rivera-Calzada, G. Waksman, Microreview: Type IV secretion systems: versatility and diversity in function. Cellular Microbiology 12, 1203-1212 (2010).

29. L. Chen, Y. Chen, D. W. Wood, E. W. Nester, A New Type IV Secretion System Promotes Conjugal Transfer in Agrobacterium tumefaciens. Journal of Bacteriology 184, 4838-4845 (2002). 
30. H. Luo, M. A. Moran, Evolutionary ecology of the marine Roseobacter clade. Microbiology and Molecular Biology Reviews 78, 573-587 (2014).

31. K. V. Korotkov, M. Sandkvist, W. G. J. Hol, The type II secretion system: biogenesis, molecular architecture and mechanism. Nature Reviews Microbiology 10, 336-351 (2012).

32. M. S. Nelson, M. J. Sadowsky, Secretion systems and signal exchange between nitrogenfixing rhizobia and legumes. Front Plant Sci 6, 491-491 (2015).

33. H. Geng, R. Belas, Molecular mechanisms underlying roseobacter-phytoplankton symbioses. Current Opinion in Biotechnology 21, 332-338 (2010).

34. P. Bartling, J. Vollmers, J. Petersen, The first world swimming championships of roseobacters-Phylogenomic insights into an exceptional motility phenotype. Systematic and Applied Microbiology 41, 544-554 (2018).

35. V. Michael, O. Frank, P. Bartling, C. Scheuner, M. Goker, H. Brinkmann, J. Petersen, Biofilm plasmids with a rhamnose operon are widely distributed determinants of the 'swim-or-stick' lifestyle in roseobacters. The ISME Journal 10, 2498-2513 (2016).

36. O. A. Soutourina, P. N. Bertin, Regulation cascade of flagellar expression in Gramnegative bacteria. FEMS Microbiology Reviews 27, 505-523 (2003).

37. R. A. Alm, P. Guerry, T. J. Trust, The Campylobacter sigma 54 flaB flagellin promoter is subject to environmental regulation. Journal of Bacteriology 175, 4448-4455 (1993).

38. C. Karlsen, S. M. Paulsen, H. S. Tunsjø, S. Krinner, H. Sørum, P. Haugen, N.-P. Willassen, Motility and flagellin gene expression in the fish pathogen Vibrio salmonicida: Effects of salinity and temperature. Microbial Pathogenesis 45, 258-264 (2008).

39. A. Guzzo, C. Diorio, M. S. DuBow, Transcription of the Escherichia coli fliC gene is regulated by metal ions. Applied and Environmental Microbiology 57, 2255-2259 (1991).

40. F. M. Cohan, Transmission in the origins of bacterial diversity, from ecotypes to phyla. Microbiology Spectrum 5, (2017).

41. B. J. Shapiro, J. Friedman, O. X. Cordero, S. P. Preheim, S. C. Timberlake, G. Szabó, M. F. Polz, E. J. Alm, Population genomics of early events in the ecological differentiation of bacteria. Science 336, 48-51 (2012).

42. H. Cadillo-Quiroz, X. Didelot, N. L. Held, A. Herrera, A. Darling, M. L. Reno, D. J. Krause, R. J. Whitaker, Patterns of gene flow define species of thermophilic Archaea. PLOS Biology 10, e1001265 (2012).

43. J.-B. Raina, V. Fernandez, B. Lambert, R. Stocker, J. R. Seymour, The role of microbial motility and chemotaxis in symbiosis. Nature Reviews Microbiology 17, 284-294 (2019).

44. L.-M. Bobay, H. Ochman, Factors driving effective population size and pan-genome evolution in bacteria. BMC Evolutionary Biology 18, 153 (2018).

45. C. Fraser, E. J. Alm, M. F. Polz, B. G. Spratt, W. P. Hanage, The bacterial species challenge: making sense of genetic and ecological diversity. Science 323, 741-746 (2009).

46. B. Charlesworth, Effective population size. Current Biology 12, R716-R717 (2002).

47. R. J. Hall, F. J. Whelan, J. O. McInerney, Y. Ou, M. R. Domingo-Sananes, Horizontal Gene transfer as a source of conflict and cooperation in Prokaryotes. Frontiers in Microbiology 11, (2020). 
48. Y. Liu, Q. Lin, J. Feng, F. Yang, H. Du, Z. Hu, H. Wang, Differences in metabolic potential between particle-associated and free-living bacteria along Pearl River Estuary. Science of The Total Environment 728, 138856 (2020).

49. P. Daste, D. Neuville, B. Victor-Baptiste, A simple procedure for obtaining clonal isolation of diatoms. British Phycological Journal 18, 1-3 (1983).

50. R. R. L. Guillard, J. H. Ryther, Studies of marine planktonic diatoms. I. Cyclotella nana Hustedt, and Detonula confervacea (cleve) Gran. Canadian Journal of Microbiology $\mathbf{8}$, 229-239 (1962).

51. M. Sapp, A. S. Schwaderer, K. H. Wiltshire, H. G. Hoppe, G. Gerdts, A. Wichels, Species-specific bacterial communities in the phycosphere of microalgae? Microbial Ecology 53, 683-699 (2007).

52. J. Zhou, M. A. Bruns, J. M. Tiedje, DNA recovery from soils of diverse composition. Applied and Environmental Microbiology 62, 316 (1996).

53. L. Hu, X. Peng, J. Zhou, Y. Zhang, S. Xu, X. Mao, Y. Gao, J. Liang, Characterizing the interactions among a dinoflagellate, flagellate and bacteria in the phycosphere of Alexandrium tamarense (Dinophyta). Frontiers in Marine Science 2, 100 (2015).

54. M. Johnson, I. Zaretskaya, Y. Raytselis, Y. Merezhuk, S. McGinnis, T. L. Madden, NCBI BLAST: a better web interface. Nucleic Acids Research 36, W5-W9 (2008).

55. F. Sievers, A. Wilm, D. Dineen, T. J. Gibson, K. Karplus, W. Li, R. Lopez, H. McWilliam, M. Remmert, J. Soding, Fast, scalable generation of high - quality protein multiple sequence alignments using Clustal Omega. Molecular Systems Biology 7, 539539 (2011).

56. S. Capellagutierrez, J. M. Sillamartinez, T. Gabaldon, trimAl: a tool for automated alignment trimming in large-scale phylogenetic analyses. Bioinformatics 25, 1972-1973 (2009).

57. L.-T. Nguyen, H. A. Schmidt, A. von Haeseler, B. Q. Minh, IQ-TREE: a fast and effective stochastic algorithm for estimating maximum-likelihood phylogenies. Molecular Biology and Evolution 32, 268-274 (2014).

58. S. Kalyaanamoorthy, B. Q. Minh, T. K. F. Wong, A. von Haeseler, L. S. Jermiin, ModelFinder: fast model selection for accurate phylogenetic estimates. Nature Methods 14, 587-589 (2017).

59. D. T. Hoang, O. Chernomor, A. von Haeseler, B. Q. Minh, L. S. Vinh, UFBoot2: Improving the ultrafast bootstrap approximation. Molecular Biology and Evolution 35, 518-522 (2018).

60. I. Letunic, P. Bork, Interactive Tree Of Life (iTOL) v4: recent updates and new developments. Nucleic Acids Research 47, W256-W259 (2019).

61. H. Wang, H. D. t. Laughinghouse, M. A. Anderson, F. Chen, E. Williiams, A. R. Place, O. Zmora, Y. Zohar, T. Zheng, R. T. Hill, Novel bacterial isolate from Permian groundwater, capable of aggregating potential biofuel-producing microalga Nannochloropsis oceanica IMET1. Applied and Environmental Microbiology 78, 14451453 (2012).

62. D. H. Parks, M. Chuvochina, D. W. Waite, C. Rinke, A. Skarshewski, P. Chaumeil, P. Hugenholtz, A standardized bacterial taxonomy based on genome phylogeny substantially revises the tree of life. Nature Biotechnology 36, 996-1004 (2018).

63. S. Nurk, A. Bankevich, D. Antipov, A. A. Gurevich, A. Korobeynikov, A. Lapidus, A. D. Prjibelski, A. Pyshkin, A. Sirotkin, Y. Sirotkin, R. Stepanauskas, S. R. Clingenpeel, T. 
Woyke, J. S. McLean, R. Lasken, G. Tesler, M. A. Alekseyev, P. A. Pevzner, Assembling single-cell genomes and mini-metagenomes from chimeric MDA products. Journal of Computational Biology 20, 714-737 (2013).

64. D. H. Parks, M. Imelfort, C. T. Skennerton, P. Hugenholtz, G. W. Tyson, CheckM: assessing the quality of microbial genomes recovered from isolates, single cells, and metagenomes. Genome Research 25, 1043-1055 (2015).

65. Y. Chen, F. Nie, S.-Q. Xie, Y.-F. Zheng, Q. Dai, T. Bray, Y.-X. Wang, J.-F. Xing, Z.-J. Huang, D.-P. Wang, L.-J. He, F. Luo, J.-X. Wang, Y.-Z. Liu, C.-L. Xiao, Efficient assembly of nanopore reads via highly accurate and intact error correction. Nature Communications 12, 60 (2021).

66. M. Kolmogorov, J. Yuan, Y. Lin, P. A. Pevzner, Assembly of long, error-prone reads using repeat graphs. Nature Biotechnology 37, 540-546 (2019).

67. R. Vaser, I. Sovic, N. Nagarajan, M. Sikic, Fast and accurate de novo genome assembly from long uncorrected reads. Genome Research 27, 737-746 (2017).

68. B. J. Walker, T. Abeel, T. Shea, M. Priest, A. Abouelliel, S. Sakthikumar, C. A. Cuomo, Q. Zeng, J. Wortman, S. K. Young, A. M. Earl, Pilon: an integrated tool for comprehensive microbial variant detection and genome assembly improvement. Plos One 9, e112963 (2014).

69. R. R. Wick, M. B. Schultz, J. Zobel, K. E. Holt, Bandage: interactive visualization of de novo genome assemblies. Bioinformatics 31, 3350-3352 (2015).

70. T. Seemann, Prokka: rapid prokaryotic genome annotation. Bioinformatics 30, 2068-2069 (2014).

71. T. Brettin, J. J. Davis, T. Disz, R. A. Edwards, S. Gerdes, G. J. Olsen, R. Olson, R. Overbeek, B. Parrello, G. D. Pusch, M. Shukla, J. A. Thomason, R. Stevens, V. Vonstein, A. R. Wattam, F. Xia, RASTtk: A modular and extensible implementation of the RAST algorithm for building custom annotation pipelines and annotating batches of genomes. Scientific Reports 5, 8365 (2015).

72. M. Kanehisa, S. Goto, S. Kawashima, A. Nakaya, S. K. S, KEGG: kyoto encyclopaedia of genes and genomes. Nucleic Acids Research 28, 27-30(24) (2000).

73. G. S. Vernikos, J. Parkhill, Interpolated variable order motifs for identification of horizontally acquired DNA: revisiting the Salmonella pathogenicity islands. Bioinformatics 22, 2196-2203 (2006).

74. D. H. Parks, C. Rinke, M. Chuvochina, P.-A. Chaumeil, B. J. Woodcroft, P. N. Evans, P. Hugenholtz, G. W. Tyson, Recovery of nearly 8,000 metagenome-assembled genomes substantially expands the tree of life. Nature Microbiology 2, 1533-1542 (2017).

75. M. Simon, C. Scheuner, J. P. Meierkolthoff, T. Brinkhoff, I. Wagnerdobler, M. Ulbrich, H. Klenk, D. Schomburg, J. Petersen, M. Goker, Phylogenomics of Rhodobacteraceae reveals evolutionary adaptation to marine and non-marine habitats. The ISME Journal 11, 1483-1499 (2017).

76. J. Rozewicki, S. Li, K. M. Amada, D. M. Standley, K. Katoh, MAFFT-DASH: integrated protein sequence and structural alignment. Nucleic Acids Research 47, W5-W10 (2019).

77. T. J. Treangen, B. D. Ondov, S. Koren, A. M. Phillippy, The Harvest suite for rapid coregenome alignment and visualization of thousands of intraspecific microbial genomes. Genome Biology 15, 524 (2014). 
78. C. Jain, L. M. Rodriguezr, A. M. Phillippy, K. T. Konstantinidis, S. Aluru, High throughput ANI analysis of $90 \mathrm{~K}$ prokaryotic genomes reveals clear species boundaries. Nature Communications 9, 5114 (2018).

79. R. C. Team, R: A language and environment for statistical computing. MSOR connections 1, 12-21 (2014).

80. A. C. Darling, B. Mau, F. R. Blattner, N. T. Perna, Mauve: multiple alignment of conserved genomic sequence with rearrangements. Genome Research 14, 1394-1403 (2004).

81. A. J. Page, C. A. Cummins, M. Hunt, V. K. Wong, S. Reuter, M. T. Holden, M. Fookes, D. Falush, J. A. Keane, J. Parkhill, Roary: rapid large-scale prokaryote pan genome analysis. Bioinformatics 31, 3691-3693 (2015).

82. C. Chen, H. Chen, Y. Zhang, H. R. Thomas, M. H. Frank, Y. He, R. Xia, TBtools: An integrative toolkit developed for interactive analyses of big biological data. Molecular Plant 13, 1194-1202 (2020).

83. L. Marquez, G. A. Morales, M. A. S. De Rodriganez, E. Almansa, F. J. Moyano, M. Diaz, Comments on the calculation of the specific growth rate in experiments with untagged individuals. Scientia Marina 79, 505-508 (2015).

84. R. P. Trenkenshu, Calculayion of the specific growth rate of microalgae. Marine Biological Journal 4, 100-108 (2019).

85. D. Pfeiffer, D. Schüler, Quantifying the benefit of a dedicated "magnetoskeleton" in bacterial magnetotaxis by live-cell motility tracking and soft agar swimming assay. Applied and Environmental Microbiology 86, e01976-01919 (2020).

86. D. Krysciak, J. Grote, M. Rodriguez Orbegoso, C. Utpatel, K. U. Förstner, L. Li, C. Schmeisser, H. B. Krishnan, W. R. Streit, RNA Sequencing analysis of the broad-hostrange strain Sinorhizobium fredii NGR234 identifies a large set of genes linked to quorum sensing-dependent regulation in the background of a traI and $n g r I$ deletion mutant. Applied and Environmental Microbiology 80, 5655 (2014).

87. E. Teira, S. Martínez-García, C. Lønborg, X. A. Alvarez-Salgado, Growth rates of different phylogenetic bacterioplankton groups in a coastal upwelling system. Environmental microbiology reports 1, 545-554 (2009).

88. Y. Sun, K. E. Powell, W. Sung, M. Lynch, M. A. Moran, H. Luo, Spontaneous mutations of a model heterotrophic marine bacterium. The ISME Journal 11, 1713-1718 (2017). 


\section{Acknowledgements}

We thank Xingqin Lin for helpful discussion. We also thank Qingyu Li for assisting

540 microalgal isolation and identification, Min Yang for helping with microalgal cell counting,

541 Fumin Yang, Qing Zhang and Huiling Chen for their help in bacteria isolation, Xiaoyu Yang for

542 assisting a preliminary experiment of the motility assays. H.W. was supported by the National

543 Natural Science Foundation of China (41676116), Key Special Project for Introduced Talents

544 Team of Southern Marine Science and Engineering Guangdong Laboratory (Guangzhou)

545 (GML2019ZD0606), and Guangdong Science and Technology Department

546 (2019A1515011139). H.L. was supported by the Hong Kong Research Grants Council General

547 Research Fund (14163917) and the Hong Kong Research Grants Council Area of Excellence

548 Scheme (AoE/M-403/16).

550 Author Contributions

551 H.L. conceptualized the work, designed this study, directed the bioinformatics analyses,

552 interpreted the data, and wrote the main manuscript. H.W. directed the sampling work, diatom

553 isolation, and roseobacter cultivation, experimental assay, and related writing, co-interpreted the

554 data, and provided comments to the manuscript. L.Q. collected the sample, performed cultivation

555 and characterized the cultures. X.F. and L.Q. performed all the bioinformatics, co-interpreted the

556 data, drafted the technical details, and prepared figures and tables. L.L. contributed to bacterial

557 isolation, and L.L. and Y.C. performed physiological assays. X.W. contributed to the

558 bioinformatics. H.Z. contributed to the discussion and provided comments to the manuscript.

\section{Conflict of Interest}


The authors declare no competing commercial interests in relation to the submitted work.

\section{Data availability}

564 The 18S rRNA gene sequences of the diatoms are available at NCBI under the accession

565 number MW494549 - MW494585. The raw genomic sequencing data and assembled genomes of

566 the 294 isolates are available at NCBI under the accession number PRJNA691705. 
A

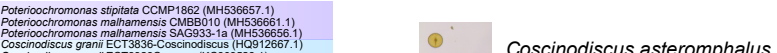

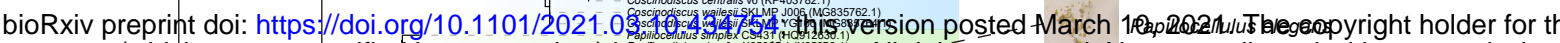

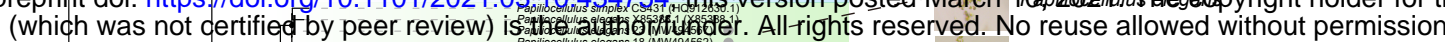

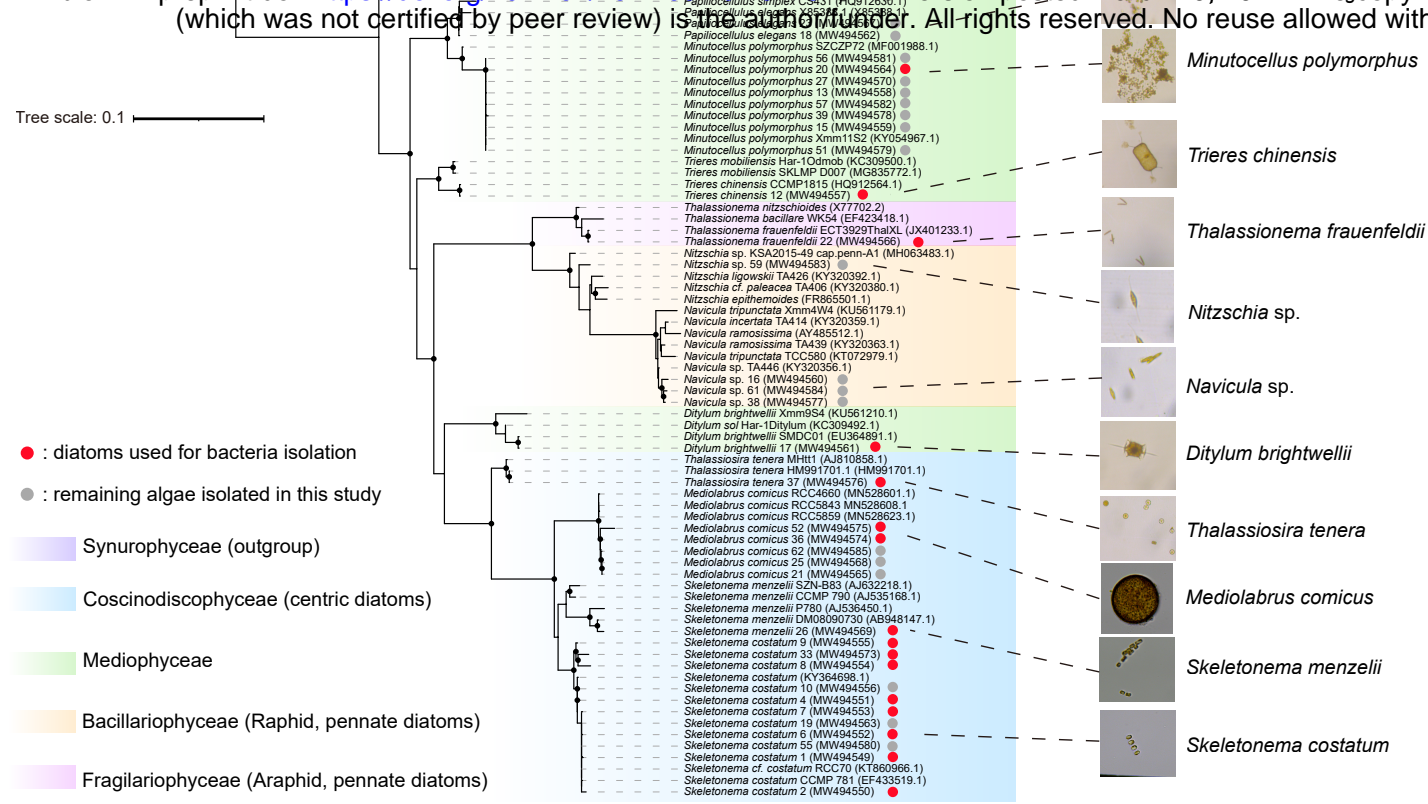

Fragilariophyceae (Araphid, pennate diatoms)

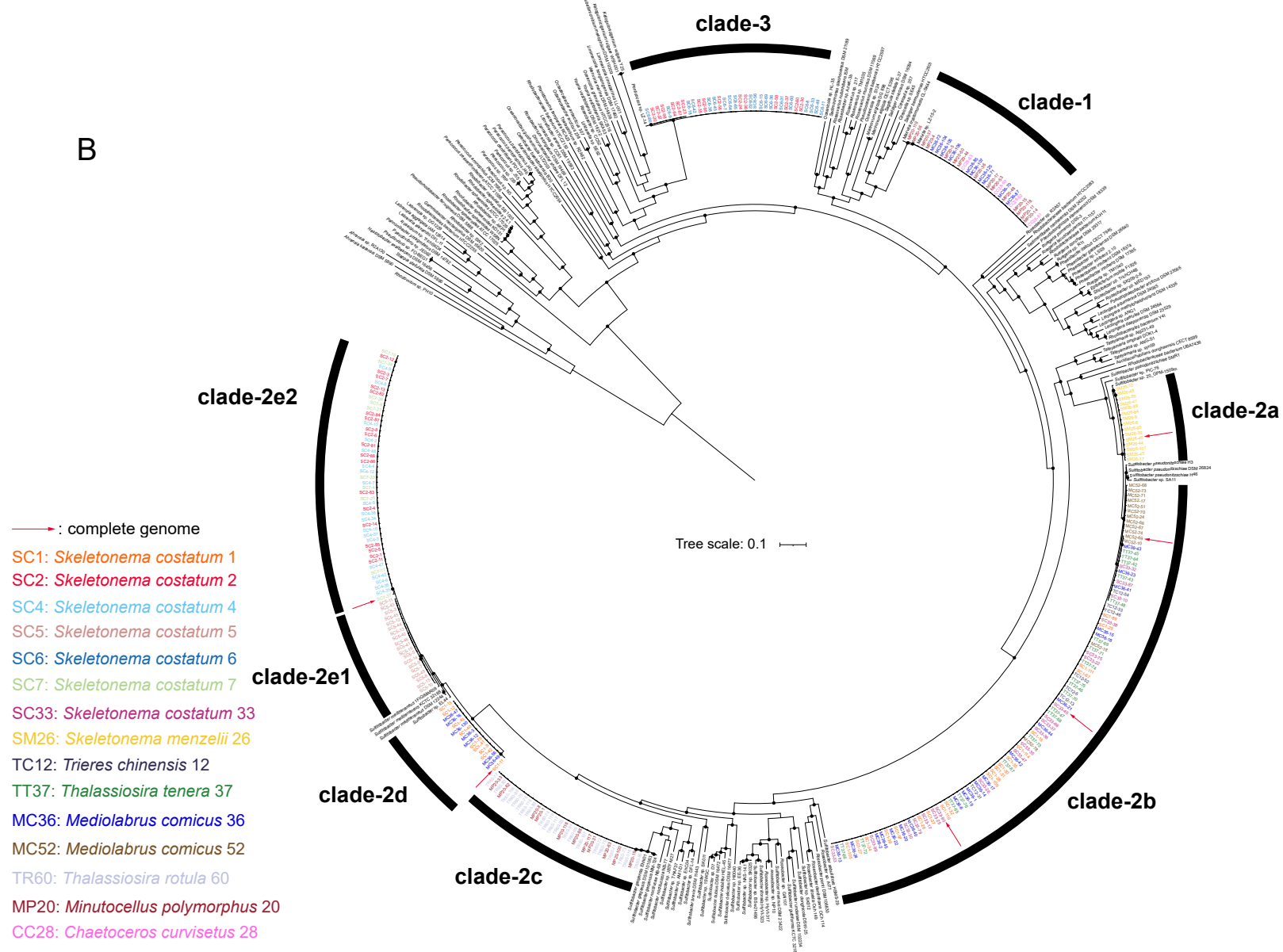

Fig 1. Phylogenetics of diatoms and Roseobacters isolated from this study. (A) Maximum likelihood (ML) tree showing the phylogenetic positions of diatoms isolated in this study. The phylogeny was inferred using IQ-TREE based on the 18S rRNA gene sequences with lengths longer than 1,600 bp. Three Synurophyceae strains were used as the outgroup. Solid circles in the phylogeny indicate nodes with ultrafast bootstrap (UFBoot) values $>95 \%$. Diatom strains used for bacteria cultivation and other isolated microalgae are marked with red and gray dots, respectively. The photos of microalgae obtained under an optical microscope are shown on the right panel. (B) ML phylogenomic tree showing the phylogenetic positions of roseobacters isolated from this study. The phylogeny was inferred using IQ-TREE based on the concatenation of 120 conserved bacterial proteins (see methods). Solid circles in the phylogeny indicate nodes with UFBoot values $>95 \%$. The associated microalgae of bacterial strains are differentiated with colors. The six complete and closed genomes are marked with red arrows. 
A

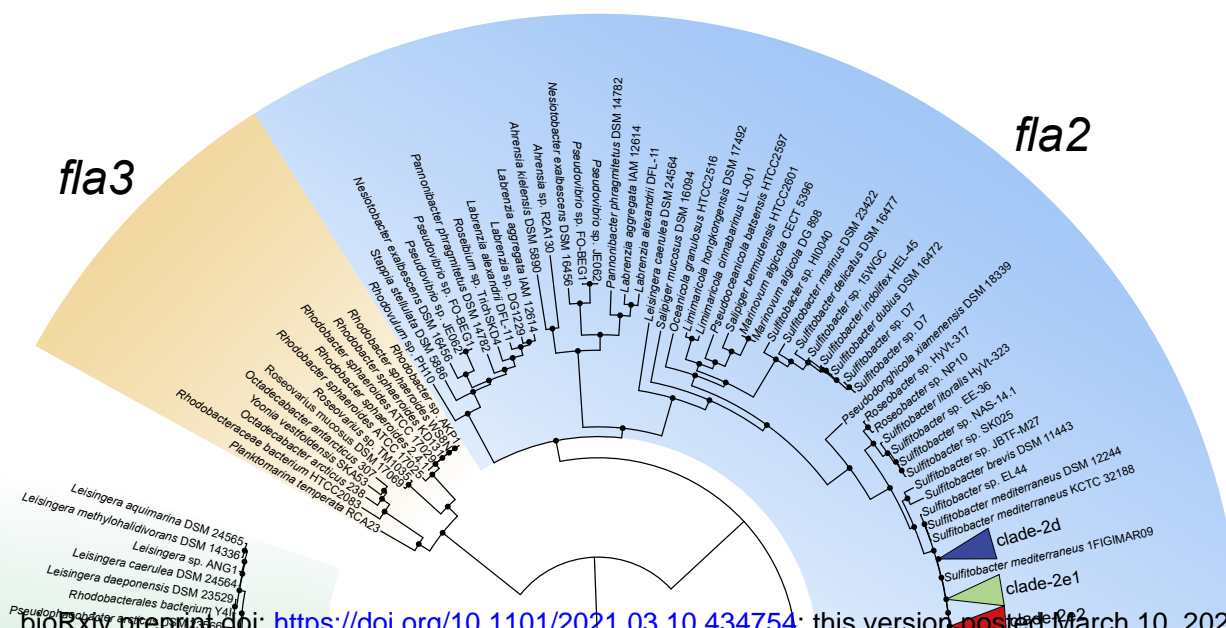

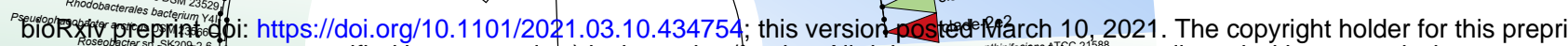

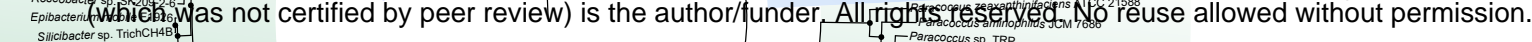
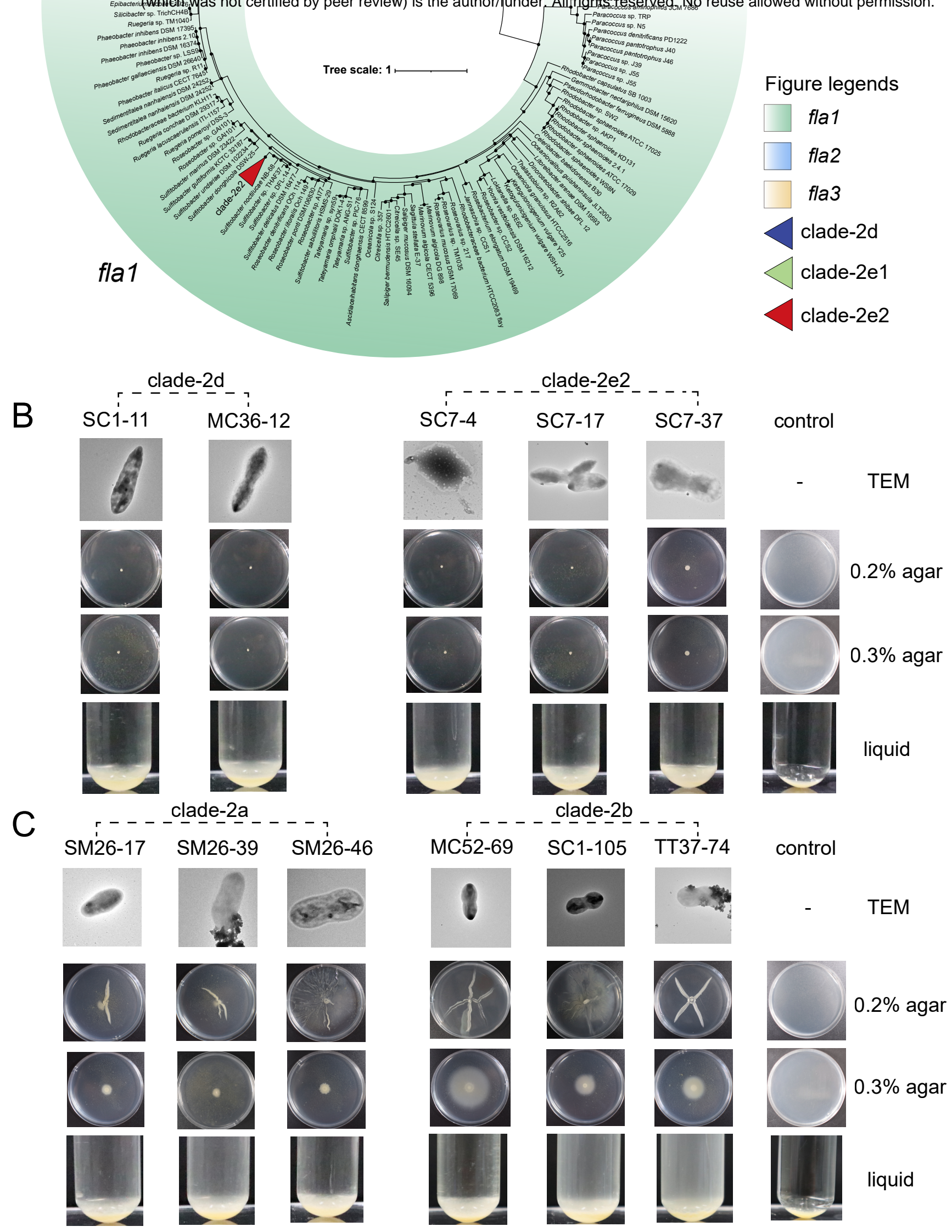

Fig. 3. The metabolic traits. (A) ML phylogenetic tree of the three homologous types of flagellar gene clusters (FGCs) found in the Roseobacter group. This phylogeny was built based on four marker flagellar proteins (FliF, Flgl, FlgH and FlhA). Solid circles in the phylogeny indicate nodes with UFBoot values $>95 \%$. $(\mathrm{B}, \mathrm{C})$ Photos showing the cellular morphology under a transmission electron microscope (TEM), motility on the $2216 \mathrm{E}$ agar plates with $0.2 \%$ or $0.3 \%$ agar, and sedimentation phenotypes in the liquid $2216 \mathrm{E}$ medium. Eleven representative strains were used in the assay, including two from clade-2d (B), three from clade-2e2 $(B)$, three from clade-2a $(C)$ and three from clade- $2 \mathrm{~b}(\mathrm{C})$. All strains from clade-2e1 were lost. 


\section{Supplementary Materials for}

\section{Metapopulation Structure of Diatom-associated Marine Bacteria}

\author{
Liping Qu, Xiaoyuan Feng, Yuerong Chen, Lingyu Li, Xiaojun Wang, Zhong Hu, Hui Wang*,
} Haiwei Luo*

*Hui Wang. Email: wanghui@stu.edu.cn

*Haiwei Luo. Email: hluo2006@gmail.com

This PDF file includes:

Supplementary Text

Figs. S1 to S8

Other Supplementary Materials for this manuscript include the following:

Data S1 to S6 


\section{Text 1. Methods}

\subsection{Isolation, identification and phylogenetic analysis of the microalgae}

One-liter of seawater ( 0.5 m below surface) was collected from the Pearl River Estuary

$621\left(113.7221^{\circ} \mathrm{E}, 21.9935^{\circ} \mathrm{N}\right)$ in January $2018(48)$, stored in a cooler $\left(4{ }^{\circ} \mathrm{C}\right)$ and transferred to the

622 laboratory within 24 hours. Single cells of microalgae were isolated from the seawater using

623 micropipettes under optical microscope following a previously described method (49). The

624 microalgal cells were then washed repeatedly using fresh axenic F/2 medium (50) to remove the

625 free-living bacteria and loosely associated bacteria around microalgal cells (51). The washed

626 microalgal cells each were inoculated in a 24-well plate containing $1 \mathrm{~mL}$ of fresh F/2 medium to

627 increase the cell density. After incubation at $20^{\circ} \mathrm{C}$ with $12 \mathrm{~h} / 12 \mathrm{~h}$ light-dark cycle at $200 \mu \mathrm{mol}$

628 photons $\mathrm{m}^{-2} \mathrm{~s}^{-1}$ for 3-5 days, the microalgal culture in each well was transferred into a conical

629 flask with $30 \mathrm{~mL}$ of fresh $\mathrm{F} / 2$ medium to increase the biomass of microalgae. In the exponential

630 growth phase, $15 \mathrm{~mL}$ of the cultures each were fixed with $1 \%$ Lugol's solution and sent to the

631 Fujian Provincial Department of Ocean and Fisheries for morphological characterization and

632 species identification. Besides, the taxonomy of microalgae was further validated using $18 \mathrm{~S}$

633 rRNA genes. The DNA was extracted from another $10 \mathrm{~mL}$ of the microalgal cultures using the

634 CTAB method (52). The 18S rRNA genes were amplified using primers (SSU-F: 5'-

635 ACCTGGTTGATCCTGCCAGT-3' and SSU-R: 5'-TCACCTACGGAAACCTTGT-3')

636 following a previous study (53) and sent for sequencing using the Sanger platform. These 18S

637 rRNA gene sequences were subjected to a maximum likelihood (ML) phylogenetic analysis

638 along with a few reference sequences, which were selected based on a preliminary BLASTn (54)

639 result against the NCBI nt database. These sequences were aligned using Clustal Omega v1.2.4

640 (55) with default parameters and trimmed using trimAl v1.4.rev15 (56) with '-automated1' 
641 option. The phylogenetic tree was constructed using IQTREE v1.6.12 (57) with the Modelfinder

642 (58) for model selection, and 1,000 ultrafast bootstrap replicates were sampled to assess the

643 robustness of the phylogeny (59). The phylogenetic tree was visualized using iTOL v5.6 (60).

\subsection{Isolation and identification of bacteria in phycosphere}

The $1 \mathrm{~mL}$ of microalgal cultures each were collected during the logarithmic growth phase

646 followed by a 10-fold serial dilution. Solid 2216E agar plates (BD Bioscience, USA) were spread

647 with $100 \mu \mathrm{L}$ of each dilution and incubated at $25^{\circ} \mathrm{C}$ for one week. Single colonies were selected

648 and repeatedly streaked on $2216 \mathrm{E}$ agar plates to isolate and purify bacterial strains initially

649 associated with microalgae. The purified bacterial strains were subjected to the colony

650 polymerase chain reaction (colony PCR) to retrieve the $16 \mathrm{~S}$ rRNA genes for taxonomy

651 identification. The $16 \mathrm{~S}$ rRNA genes were amplified using the universal primers $27 \mathrm{~F}$ and $1492 \mathrm{R}$

652 as described previously (61) and were partially ( $700 \mathrm{bp})$ sequenced using $27 \mathrm{~F}$ on the Sanger

653 platform at Invitrogen Trading (Shanghai) Co., Ltd. Their taxonomy was inferred using BLASTn

654 on the NCBI website, and a total of 294 strains with top BLAST hit to the Roseobacter group

655 were kept for genome sequencing.

1.3 Genome sequencing, assembly and annotation

The genomic DNA of 294 Roseobacter genomes was extracted using the Bacterial Genome DNA Rapid Extraction Kit (Guangzhou Dongsheng Biotech Co., Ltd.) and sequenced at the

659 Beijing Genomics Institute (BGI, China) using the Illumina Hiseq xten PE150 platform. Raw

660 reads were quality trimmed using Trimmomatic v0.39 (62) with options

661 'SLIDINGWINDOW:4:15 MAXINFO:40:0.9 MINLEN:40' and assembled using SPAdes 662 v3.10.1(63) with '-careful' option. Only contigs with length $>1,000$ bp and sequencing depth > 
$6635 x$ were retained. Genome completeness, contamination, and strain heterogeneity (Table S3)

664 were calculated using CheckM v1.1.2 (64).

665 Six isolates (SM26-46, SC33-45, SC33-90, MC52-69, SC1-11 and SC7-37) were

666 additionally sequenced with the Nanopore platform (Nextomics Biosciences Co., Ltd.) to retrieve

667 complete and closed genomes. The mismatches between Nanopore and Illumina reads were

668 reconciled according to the following procedure. Raw reads of the Nanopore sequencing were

669 first corrected by Necat v0.0.1 (65) with 'PREP_OUTPUT_COVERAGE=100

670 CNS_OUTPUT_COVERAGE=50' parameters. The polished reads were then assembled using

671 Flye v2.6 (66) with the '--plasmids' parameter. The initial assemblies were corrected twice using

672 the polished Nanopore sequencing reads by Racon v1.4.13 (67) with '-m 8 -x -6 -g -8 -w 500'

673 options and five times using the Illumina sequencing reads by Pilon v1.23 (68) with default

674 parameters. The Bandage v0.8.1 (69) was used to check whether the final assembled

675 chromosomes and plasmids were closed, which showed that the chromosome and plasmids in all

676 six genomes are closed except the plasmid 2b_P2 in MC52-69 and plasmid 2b_P5 in SC33-90

677 (Table S4).

678 Protein-coding genes were identified using Prokka v1.14.6 (70) with default parameters, and 679 their functions were annotated using online RAST (71) and KEGG server (72). Genomic islands 680 were predicted using Alien_hunter v1.7 (73) with default parameters.

An ML phylogenetic tree was constructed based on 120 conserved bacterial genes (74) at the 683 amino acid level to identify the phylogenetic position of 294 sequenced Roseobacter genomes.

684 Other reference Roseobacter genomes included in the phylogeny were used following previous 685 studies $(35,75)$. The 120 conserved proteins each were aligned using MAFFT v7.222 (76) with 
686 default parameters and trimmed using trimAl with '-resoverlap 0.55 -seqoverlap 60’ options. The

687 trimmed alignments were linked together to form a super-alignment for each genome. The

688 phylogenetic tree was constructed using IQTREE v1.6.12 (57) with the Modelfinder (58) for

689 model selection, and a total of 1,000 ultrafast bootstrap replicates were sampled to assess the

690 robustness of the phylogeny (59). The phylogenetic tree was visualized using iTOL v5.6 (60).

\subsection{Plasmid identification for the clade-2e1 members}

Since a closed genome was not available to the clade-2e1, we used the following procedure

693 to detect whether a contig is part of the chromosome or plasmid. Contigs of clade-2e1 genomes

694 were aligned to the complete and closed genome of clade-2e2 (SC7-37) using Parsnp v1.2 (77).

695 The contig was considered to be located on the chromosome or plasmid if $>80 \%$ region of this

696 contig was aligned to the chromosome or plasmid of SC7-37. The remaining contigs were

697 considered as unassigned.

\subsection{Population genomics analyses}

The whole-genome average nucleotide identity (ANI) was calculated using FastANI v1.3

700 (78) to assess the genomic sequence similarity within and between clades. Besides, single

701 nucleotide polymorphisms (SNPs) were identified using Parsnp v1.2 (77) with default

702 parameters, and the SNP density was calculated using $10 \mathrm{Kbp}$ sliding windows and plotted with

703 custom scripts in R v3.6.1 (79). To investigate the genomic structural variation between clades,

704 two complete and closed genomes (SC1-11 in clade-2d and SC7-37 in clade-2e2) were aligned

705 using Mauve v2015-02-26 (80) with default parameters and the segment arrangement of the

706 chromosome and the plasmid was visualized using Mauve with the 'Min LCB weight' parameter

707 around 2,000. A similar comparison was also performed for two complete and closed genomes 
708 from clade-2a (SM26-46) and clade-2b (SC33-45), and for three complete and closed genomes

709 within clade-2b (SC33-45, SC33-90 and MC52-69), respectively.

710 The clustering of accessory genes within clade was used to investigate whether the

711 association with different hosts caused the differentiation of roseobacters at the genome content

712 level. Orthologous gene families (OGs) were predicted using Roary v3.13.0 (81) with default

713 parameters for genomes of the eight clades separately. The presence/absence pattern of accessory

714 OGs was summarized as a binary matrix. The Euclidean distance of each pair of genomes was

715 calculated using TBtools v1.0695 (82), and then genomes were clustered with the 'complete'

716 method and visualized using TBtools.

\subsection{Co-cultivation of an axenic diatom culture and the Roseobacter isolates}

The 11 Roseobacter representatives each were co-cultured with an axenic diatom culture

Phaeodactylum tricornutum CCMP2561 to verify the effect of these roseobacters on the

720 microalgal growth. The axenic microalgal culture was obtained from the Institute of

721 Hydrobiology, Chinese Academy of Sciences, Wuhan, China. The diatom was inoculated in

722 axenic $\mathrm{F} / 2$ medium at $20^{\circ} \mathrm{C}$ with $12 \mathrm{~h} / 12 \mathrm{~h}$ light-dark cycle at $200 \mu$ mol photons $\mathrm{m}^{-2} \mathrm{~s}^{-1}$. The

723 microalgal cells were counted three times each day using the Beckman Coulter Z2 (Beckman

724 Coulter Inc., America) until they reached the logarithmic growth phase. The initial cell

725 concentration of the microalgae used for growth assay was about $10^{6}$ cells $\mathrm{mL}^{-1}$.

726 The 11 Roseobacter strains each were inoculated in $5 \mathrm{~mL}$ of $2216 \mathrm{E}$ liquid medium and

727 incubated at $25^{\circ} \mathrm{C}$ with $150 \mathrm{rpm}$ shaking until the $\mathrm{OD}_{600}$ reached $0.6-0.7$. For each strain, $3 \mathrm{~mL}$

728 of bacterial culture was centrifuged for $1 \mathrm{~min}$ at $12,000 \mathrm{rpm}$, and pellets were washed three times

729 with sterile seawater and re-suspended in $100 \mu \mathrm{L}$ of sterile seawater. 
The $100 \mu \mathrm{L}$ of bacterial suspension was inoculated in $30 \mathrm{~mL}$ of axenic microalgal culture as

731 the experimental group, and another $30 \mathrm{~mL}$ of the axenic microalgal culture without bacterial

732 inoculations were used as the negative control. Three replicates were set for all control and

733 experimental groups. The microalgal cells were counted in triplicate at the third day of the co-

734 culture experiment. The specific growth rate of the microalgae ( $\mu_{\mathrm{c}}$ for the control group and $\mu_{\mathrm{e}}$

735 for the experimental groups) over three days was calculated as:

$$
\mu=\left(\ln B_{2}-\ln B_{1}\right) /\left(t_{2}-t_{1}\right)
$$

where $B_{1}$ and $B_{2}$ were the cellular density (concentration) in the culture at $t_{1}(0 d)$ and $t_{2}(3$

\subsection{Phylogenetic analysis for flagellar gene clusters}

Three homologous and phylogenetically distinguishable types of flagellar gene cluster

742 (FGC) have been identified in the Roseobacter group (35). While none of them were identified in

743 closely related clade-2a and clade-2b members, its homologs were found in clade-2d, clade-2e1

744 and clade-2e2 members. Next, phylogenetic analysis was performed for all the 83 genomes from

745 the latter three clades to identify their FGC type. Four flagellar marker genes $(f l i F, f l g H, f l g I$, and

$746 f(h A)(35)$ were aligned at the amino acid level using MAFFT v7.222 (76) with default

747 parameters and trimmed using trimAl with '-resoverlap 0.55 -seqoverlap 60' options. The

748 trimmed alignments were concatenated and a phylogenetic tree was constructed using IQTREE

749 v1.6.12 (57) with the Modelfinder (58) function. A total of 1,000 ultrafast bootstrap replicates

750 were sampled to assess the robustness of the phylogeny (59), and the phylogenetic tree was

751 visualized using iTOL v5.6 (60). 


\subsection{Motility assay}

Eleven roseobacters were used in the motility assay, including three strains in clade-2a, three

754 in clade-2b, two in clade-2d, and three in clade-2e2. The clade-2e1 was not included because all

755 strains in this clade were lost. The 11 strains each were inoculated in $5 \mathrm{~mL}$ of $2216 \mathrm{E}$ liquid

756 medium and incubated at $25^{\circ} \mathrm{C}$ with $150 \mathrm{rpm}$ shaking until the optical density value at $600 \mathrm{~nm}$

$757\left(\mathrm{OD}_{600}\right)$ reached 0.6-0.7. The flagella and pili were observed under a transmission electron

758 microscope (TEM; JEM-F200, Japan), and the motility of representative strains was tested on

759 solid and liquid medium.

760 Aliquots $(6 \mathrm{~mL})$ of liquid culture for each bacterial strain was centrifuged at 1,000 rpm for

$76110 \mathrm{~min}$. The pellets were rinsed with axenic water 2-3 times. Pellets were fixed overnight with 1

$762 \mathrm{~mL}$ of $2.5 \%$ glutaraldehyde solution at $4{ }^{\circ} \mathrm{C}$. Fixed cells were resuspended with axenic water and

763 stabilized on the 200-mesh copper grid (Beijing Zhongjingkeyi Technology Co., Ltd) with the

764 carbon support film. Stabilized cells were stained with $2 \%$ sodium phosphotungstate solution for

$765 \quad 1-2$ min and observed by TEM.

766 The motility of the 11 strains were tested on soft agar plates with $0.2 \%$ and $0.3 \%$ agar (w/v)

767 following previously study $(34,85)$. Soft agar plates were point inoculated with $3 \mu \mathrm{L}$ of bacterial

768 pre-cultures and incubated at $25^{\circ} \mathrm{C}$ for $6 \mathrm{~d}$. The motility was also tested using sedimentation

769 assays by inoculating $100 \mu \mathrm{L}$ of bacterial cultures in $5 \mathrm{~mL}$ of fresh $2216 \mathrm{E}$ liquid medium and

770 incubated at room temperature for $24 \mathrm{~h}$ without shaking (86). 


\section{Text 2. Supplemental result}

773 High genetic similarities of within-clade members are not due to clonal replication during

774 laboratory cultivation

775 Considering that all members of each clade showed extremely high genetic identity at the

776 DNA sequence level, it is possible that the collected isolates can be a mix of cells each

777 representing a distinct genotype originally inhabiting the wild environment and cells replicated

778 clonally during the laboratory cultivation of the microalgae. The latter situation needs to be

779 considered since roseobacter isolation was performed after the one-month incubation of

780 microalgae and since roseobacters divide approximately once per day (87). It is important to

781 differentiate these two scenarios because the inference of population structure could be biased if

782 laboratory clones dominated our collections. Here, we provide several lines of evidence against

783 the laboratory replication hypothesis.

784 If all members of a clade would have been laboratory replicates from a single ancestor, we

785 asked whether the observed SNPs could be explained by mutations during the period of

786 laboratory cultivation of the diatoms. If a simple assumption is made that each SNP site was

787 caused by a mutation, then the expected number of mutations $(S)$ can be estimated based on the

788 spontaneous mutation rate $(\mu)$, bacterial growth rate or number of cell divisions per day $(G)$, the

789 alignment length $(L)$, laboratory cultivation time $(T)$ and number of genomes under comparison

$790(N)$, according to the following equation:

$$
S=\mu * G * L * T * N
$$

792 To make it simple, we used the mutation rate of the model Roseobacter strain, Ruegeria

793 pomeroyi DSS-3 $\left(\mu=1.39 \times 10^{-10}\right.$ per nucleotide per generation) (88) and the doubling time of

794 wild roseobacters ( $G=$ approximately one generation per day) (87). Given that $L=4.34,4.84$, 

clade-1, clade-2a, clade-2b, clade-2c, clade-2d, clade-2e1, clade-2e2 and clade-3, respectively,

797 the expected number of mutations of these clades are $0.53,0.30,1.82,0.52,0.24,0.31,0.75$ and

798 0.63, respectively, under 30 days of laboratory cultivation of the diatoms. Since the expected

799 numbers of mutations are all below one except clade-2b but there are a few dozen SNPs in six of

800 the eight clades (Table S5), the observed SNPs cannot arise from mutations during the laboratory 801 cultivation period.

802 One may argue that some of the SNPs could result from sequencing error. While it is 803 difficult to distinguish between mutations and sequencing errors for singleton SNPs, presence of 804 non-singleton SNPs (the rare variant found in at least two genomes) is convincing evidence 805 against sequencing errors owing to the extremely low chance of having sequencing errors at the 806 same sites. We identified non-singleton SNPs in six out of the eight clades (Table S5). In 807 particular, all sequenced genomes from clade-1 and clade-2a each exhibited a unique 808 combination of the non-singleton SNPs, suggesting that none of them represent laboratory clones 809 (Table S5). For members from clade-2b, clade-2e1, clade-2e2 and clade-3, we identified 26, 14, 8109 and 14 unique combinations of the non-singleton SNPs in 100, 19, 48 and 35 genomes,

811 respectively, suggesting that at least a sizable number of the members in these clades represent

812 the wild genotypes. In the case of the remaining two clades, clade- $2 \mathrm{c}$ and clade-2d, no non-

813 singleton SNPs were found in the 32 and 16 members, respectively.

814 It is important to note that the SNP analysis is restricted to the core DNA shared by all 815 members of a clade, and members without displaying biologically meaningful SNPs in their core 816 genomes may differ in their accessory genomes. We therefore asked whether genomes from each 817 clade each harbor a unique combination of accessory genes (Table S5). However, this analysis is 
818 compromised by the incomplete genome assembly of reads derived from Illumina sequencing.

819 This issue is mitigated, but not eradicated, when the genes missing in the complete and closed

820 genome are used. For the four clades (clade-2a, clade-2b, clade-2d and clade-2e2) with at least

821 one complete and closed genome, all of genomes each exhibit a unique combination of accessory

822 genes (Table S5), which is evidence against the laboratory clonal replication hypothesis.

823 A higher-level marker to discriminate between genomes is gene order and genome

824 rearrangement, which is best characterized using complete and closed genomes. We therefore

825 closed three genomes (SC33-45, SC33-90 and MC52-69) from the same clade (clade-2b) each

826 with additional sequencing by Nanopore (Table S4). Particularly, two of them (SC33-45 and

827 SC33-90) are barely differentiated at the accessory genome content level (Fig. 2A), further

828 highlighting the potential value of the genome rearrangement analysis. These three genomes

829 (SC33-45, SC33-90 and MC52-69) differ in chromosomal DNA length (3,836,086 bp, 3,834,406

830 bp and 3,850,659 bp, respectively), and one DNA inversion event has occurred on their

831 chromosome involving an orthologous DNA segment varying in their length (4644 bp, $4648 \mathrm{bp}$

832 and 1164 bp, respectively; Fig. S2). They possess seven homologous plasmids, of which five are

833 closed for all three genomes and thus are useful for comparison. While these five closed

834 plasmids each have conserved gene order, they differ slightly in their lengths. Here are

835 assembled lengths of these five closed plasmids in the three isolates: 2b_P1 $(82,238$ bp, 82,229

836 bp and 82,397 bp), 2b_P3 (290,592 bp, 290,600 bp and 290,590 bp), 2b_P4 (281,159 bp,

837281,160 bp and 281,166 bp), 2b_P6 (210,913 bp, 210,913 bp and 210,915 bp), and 2b_P7

$838(110,566 \mathrm{bp}, 110,566 \mathrm{bp}$ and $110,565 \mathrm{bp})$.

839 Taken together, the available patterns of non-singleton SNPs in the core genomes, the strain-

840 specific gene combination in the accessory genomes, the length of chromosomal DNA and 
841 plasmid DNA, and strain-specific order of genomic fragments strongly favor the hypothesis that

842 most of the analyzed roseobacters each represents a distinct genotype originally from the wild

843 environment.

844 


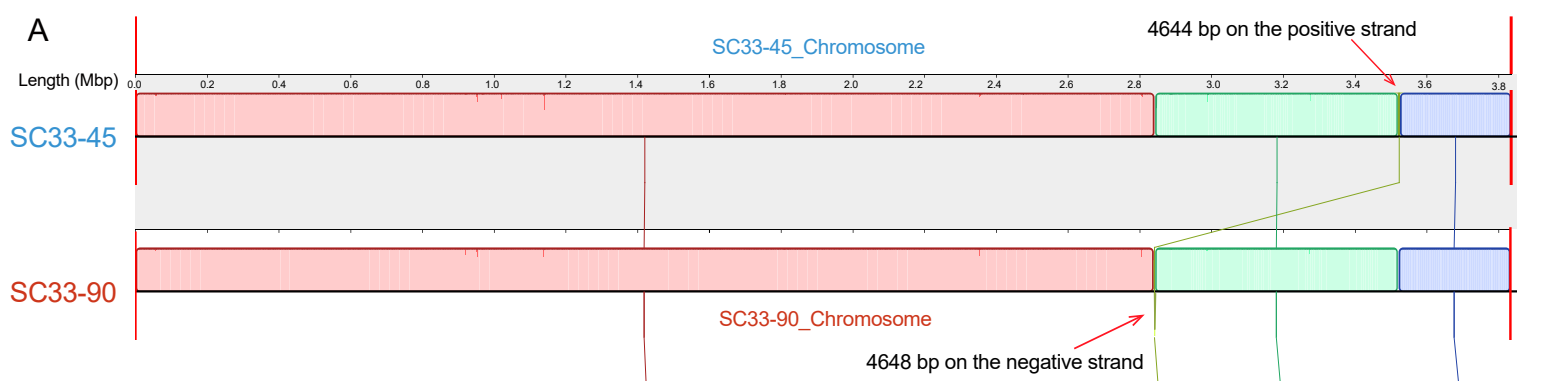

MC52-69
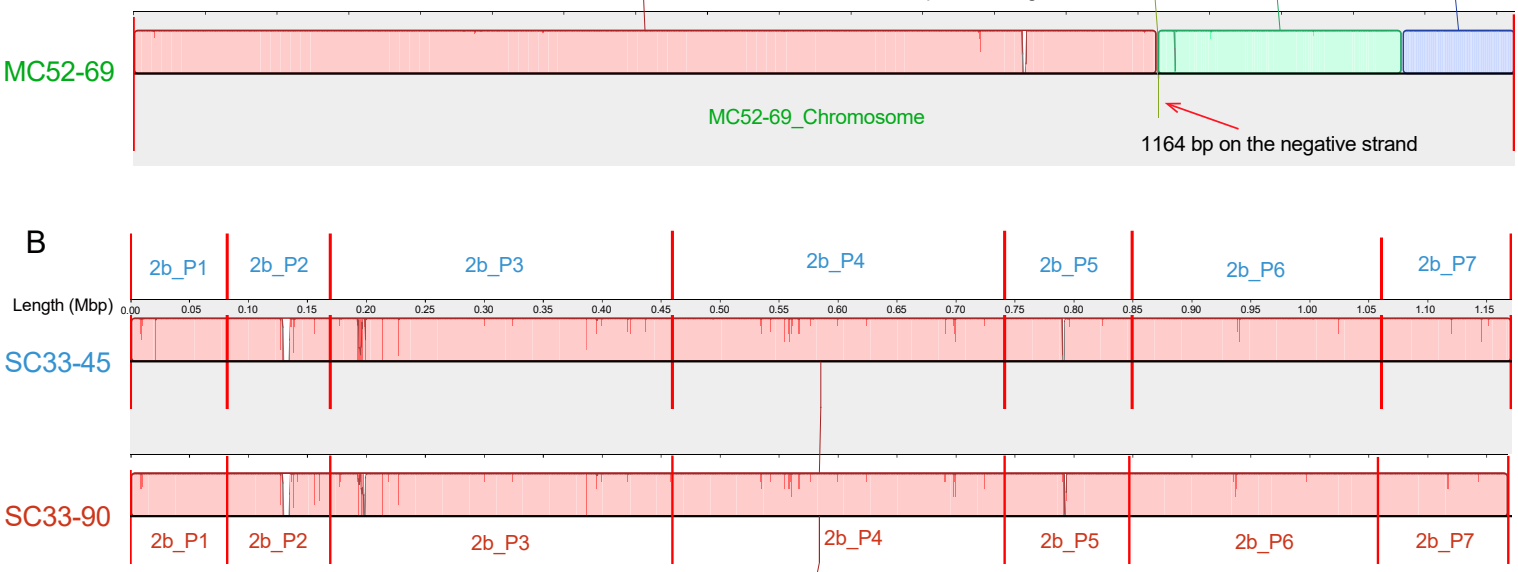

MC52-69

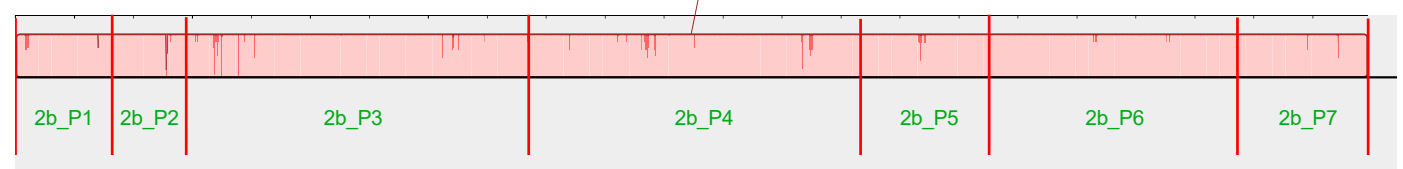

I: The boundaries of chromosome and plasmids $\quad P$ : plasmid $\quad 2 b$ : clade-2b

Fig. S2. The genome arrangement of (A) chromosome and (B) plasmids of the three complete and closed genomes (MC52-69, SC33-45 and SC33-90) in clade-2b. Homologous regions shared by the three genomes are represented using locally collinear blocks (LCBs) with connected lines. The minimum LCB weight is 3485 , which represents the minimum number of matching nucleotides identified in all LCBs. A similarity profile is shown within each LCB, and the height of the similarity profile represents the conservation level of the alignment. LCBs above and below the centerline represent genomic regions on the forward and reverse strand, respectively. The boundaries of replicons (chromosome and plasmids) are represented by red vertical lines. 
A Within clade-2e1 Reference genome: SC5-10 (clade-2e1)

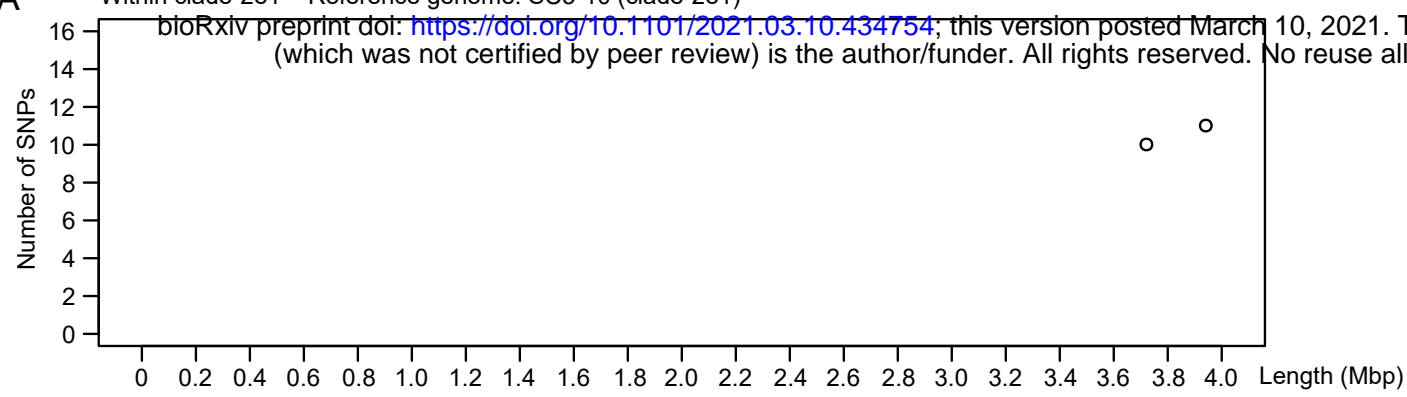

B Within clade-2e2 Reference genome: SC7-37 (clade-2e2)

13.65 Mbp

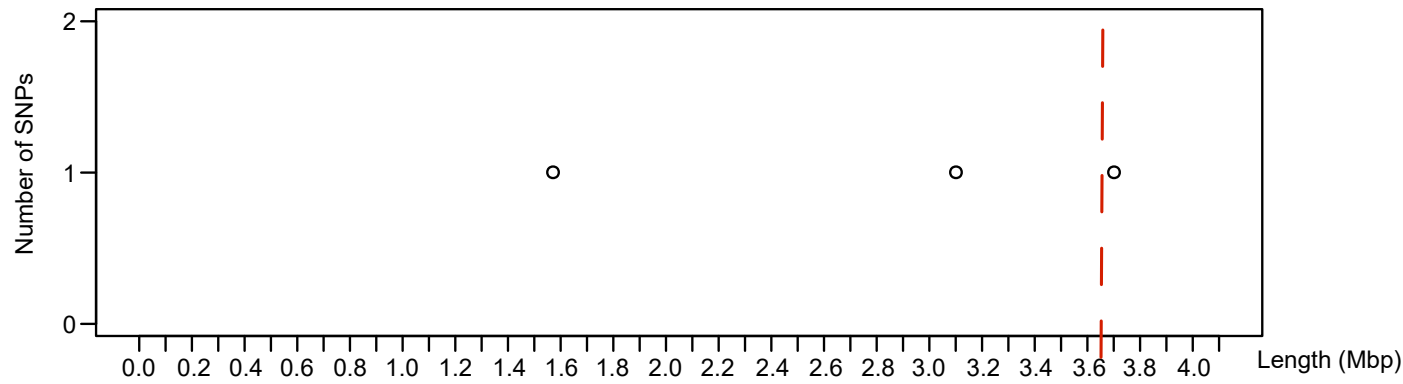

C
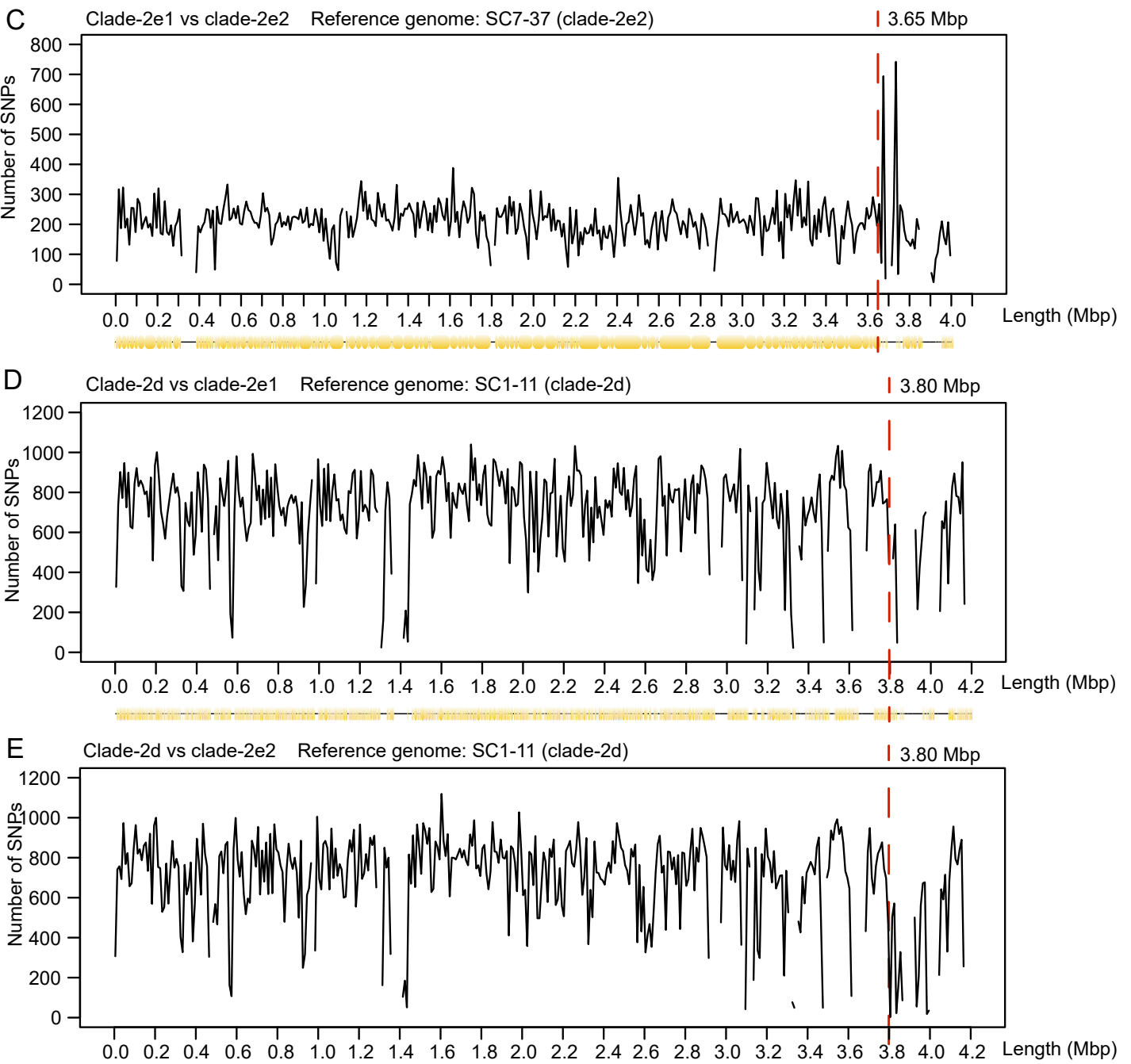

Window size: $10 \mathrm{Kbp}$

Fig. S4. The density of non-singleton SNPs (A) within clade-2e1, $(B)$ within clade-2e2, (C) between clade-2e1 and clade-2e2, (D) between clade-2d and clade-2e1, and (E) between clade-2d and clade-2e2. The SNP density of clade-2d is not shown because no SNP was found within this clade. The SNPs density was calculated with a window size of $10 \mathrm{Kbp}$. The aligned region was shown at the bottom of each panel. The boundary between chromosome and plasmid is shown using a red vertical dotted line. 

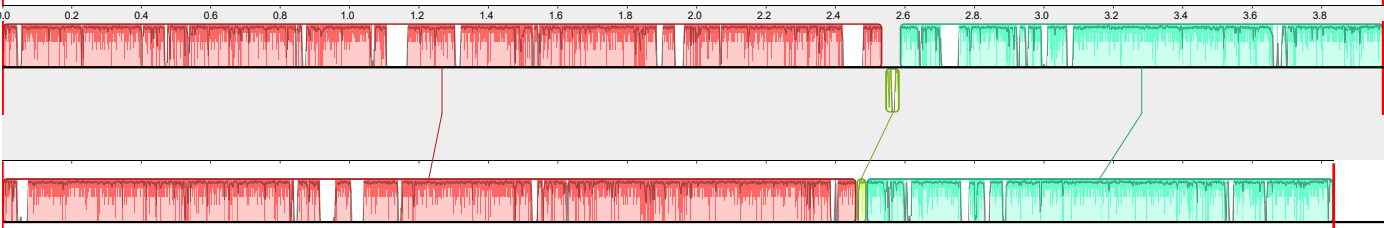

\section{SC33-45_Chromosome}

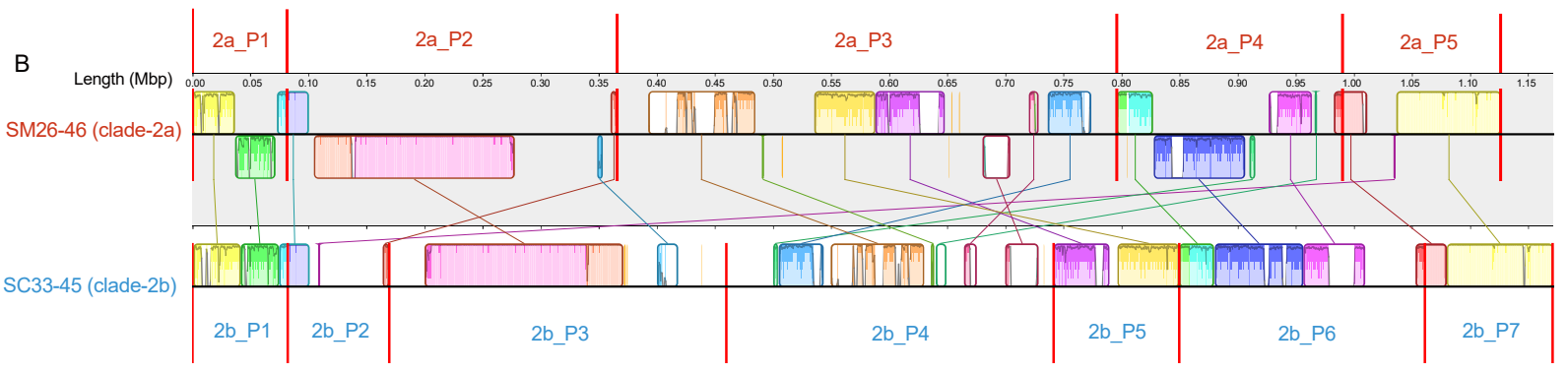

| : The boundaries of chromosome and plasmids

P: plasmid

2a: clade-2a

$2 b$ : clade- $2 b$

Fig. S5. The genome arrangement of (A) chromosome and (B) plasmids of the two complete genomes in clade-2a (SM26-46) and clade-2b (SC33-45). Homologous regions shared by the two genomes are represented using locally collinear blocks (LCBs) with connected lines. The minimum LCB weight is 2249 , which represents the minimum number of matching nucleotides identified in all LCBs. A similarity profile is shown within each LCB, and the height of the similarity profile represents the conservation level of the alignment. LCBs above and below the centerline represent genomic regions on the forward and reverse strand, respectively. The boundaries of replicons (chromosome and plasmids) are represented by red vertical lines. 

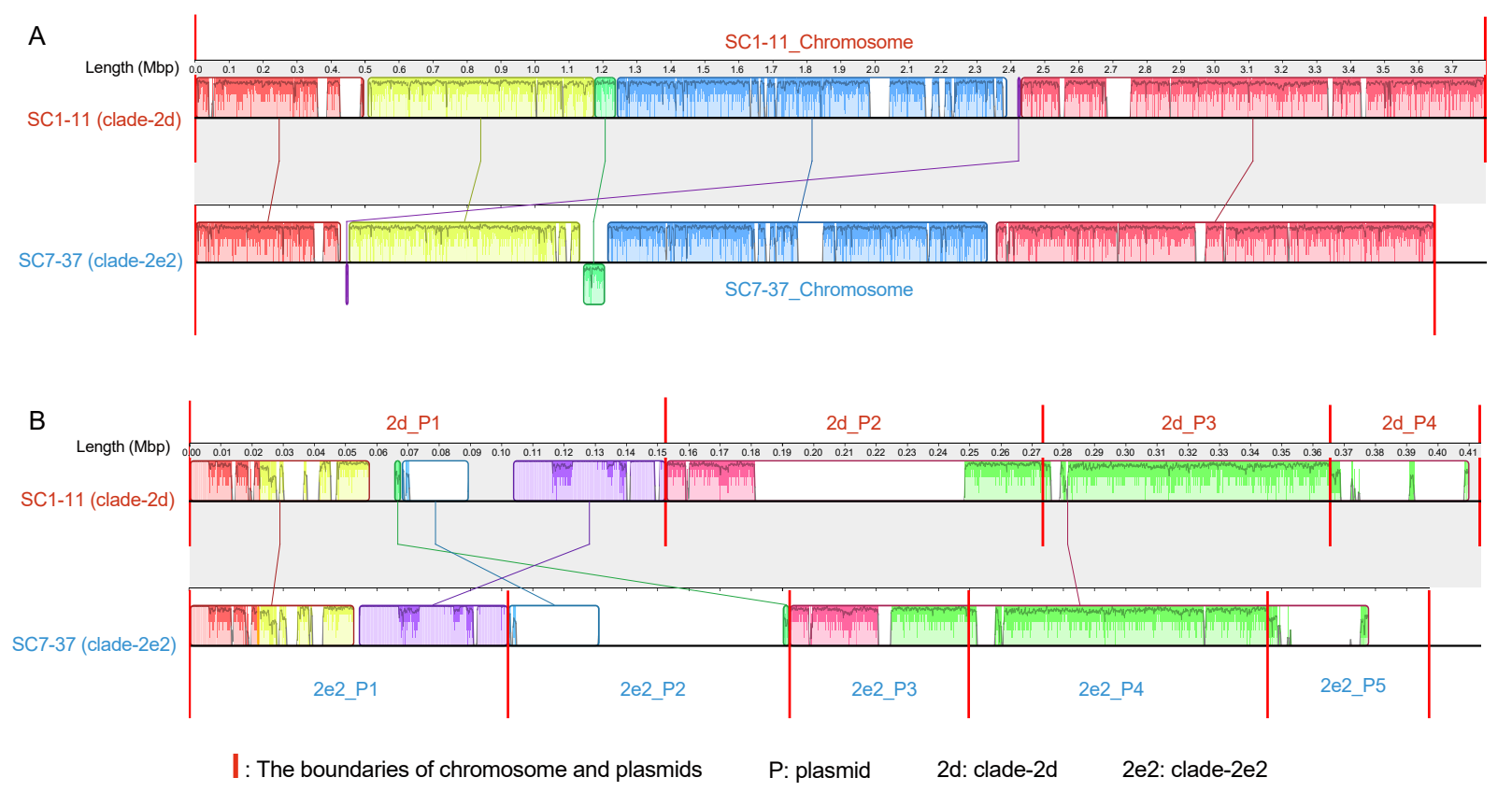

Fig. S6. The genome arrangement of (A) chromosome and (B) plasmids of the two complete genomes from clade-2d (SC1-11) and clade-2e2 (SC7-37). Homologous regions shared by the two genomes are represented using locally collinear blocks (LCBs) with connected lines. The minimum LCB weight is 2209 , which represents the minimum number of matching nucleotides identified in all LCBs. A similarity profile is shown within each LCB, and the height of the similarity profile represents the conservation level of the alignment. LCBs above and below the centerline represent genomic regions on the forward and reverse strand, respectively. The boundaries of replicons (chromosome and plasmids) are represented by red vertical lines. 


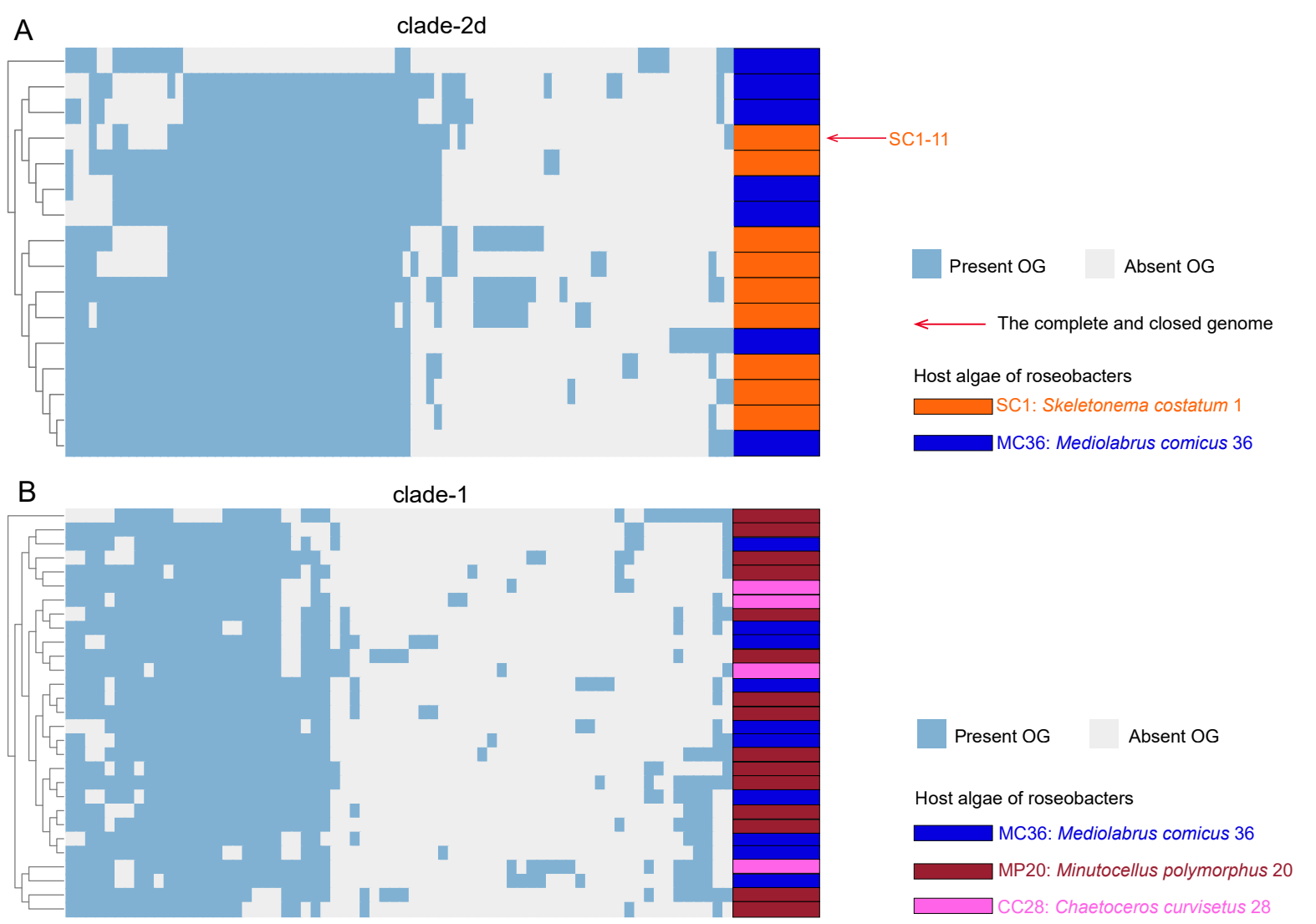

Fig. S7. The clustering of accessory genes in the genomes of clade-2d (A) and clade-1 (B). The dendrogram of genome clustering was generated based on the presence and absence of their orthologous gene families (OGs), which are colored in blue and gray, respectively. The associated microalgae of bacterial strains are differentiated with colors. The complete and closed genome is marked with a red arrow. 
The specific growth rate of microalgae $(\mu)$

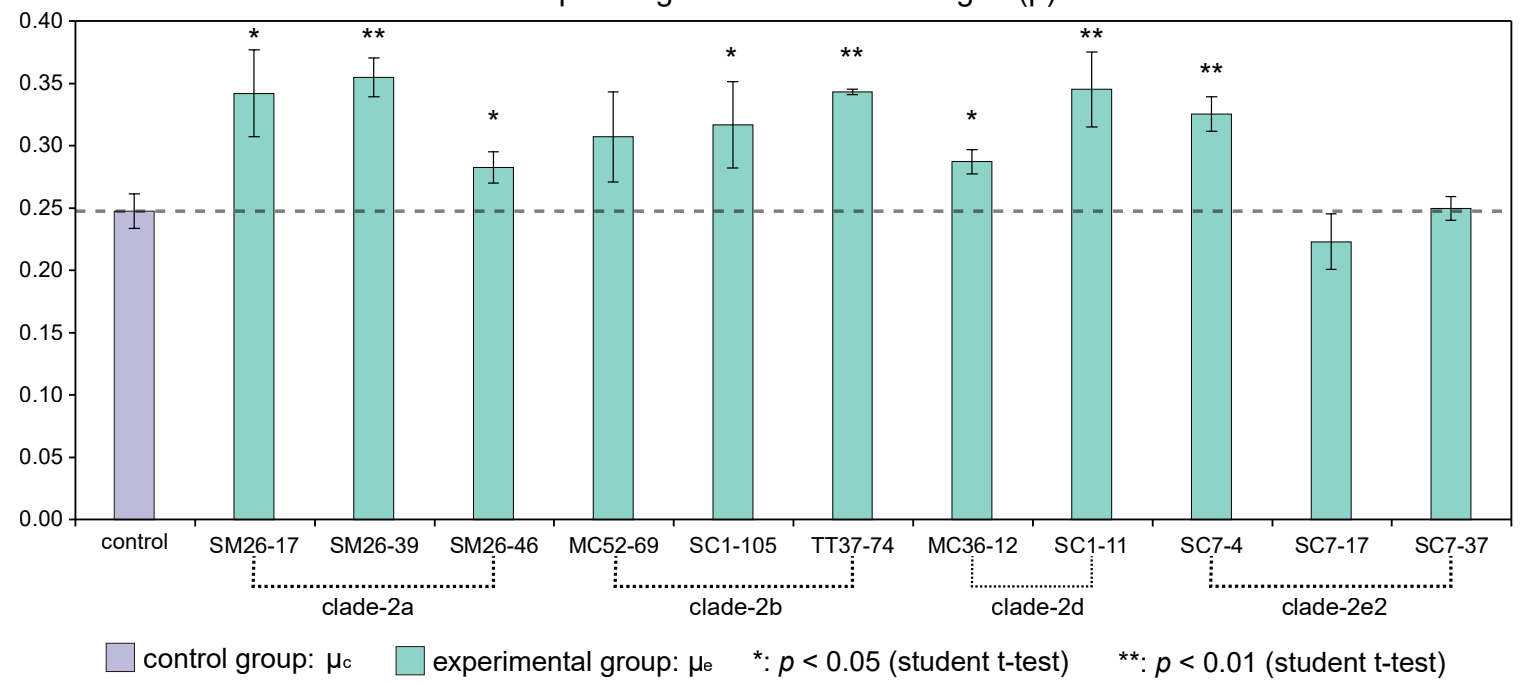

Fig. S8. The specific growth rates of microalgal strains after three days of co-culture. The microalgal specific growth rate in the control groups $\left(\mu_{\mathrm{c}}\right)$ and experimental groups $\left(\mu_{\mathrm{e}}\right)$ are shown in purple and green columns, respectively. The significance level $p<0.05$ and $p<0.01$ compared to the control group is shown using ${ }^{*}$ and ${ }^{* *}$, respectively. 